\title{
PRODUCTION THEORY: ACCOUNTING FOR FIRM HETEROGENEITY AND TECHNICAL CHANGE*
}

\author{
Giovanni Dosi ${ }^{\dagger}$ \\ MARCO GRAZZI \\ Luigi MARENGo ${ }^{\S}$ \\ Simona SeTtepanella
}

The paper presents a new framework to assess firm level heterogeneity and to study the rate and direction of technical change. Building on the analysis of revealed short-run production functions by Hildenbrand (1981), we propose the (normalized) volume of the zonotope composed by vectors-firms as indicator of inter-firm heterogeneity. Moreover, the angles that the zonotope's main diagonal form with the axes provide a measure of the rates and directions of productivity change. The proposed framework also accounts for n-inputs and m-outputs and, crucially, the measures of heterogeneity and technical change do not require many of the standard assumptions from production theory.

\section{Introduction}

In recent years an extremely robust evidence regarding firm- and plantlevel longitudinal microdata has highlighted striking and persistent heterogeneity across firms operating in the same industry. A large body of research from different sectors in different countries (cf. Baily et al. 1992, Baldwin and Rafiquzzaman 1995, Bartelsman and Doms 2000, Disney et al. 2003, Dosi 2007, Syverson 2011, among many others) documents the

*The statistical exercises which follow would not have been possible without the unmeasurable help of the Italian Statistical Office (ISTAT) and in particular of Roberto Monducci and Stefano Menghinello. Comments by Cinzia Daraio, Werner Hildenbrand, Stefano Marmi, Anwar Shaikh and two anonymous referees are gratefully acknowledged. In particular we want to thank the editor of the Journal of Industrial Economics, Chad Syverson, for his support and insightful suggestions. We also thank the participants to the conferences of the Italian Economic Society (SIE), Bologna, October 2013; Jornadas de Economia Industrial, Segovia, September 2013 and to the faculty seminars at the University of Berkeley and Notre Dame (USA), Bologna (Italy) and UCL (UK). Finally, let us thank Federico Ponchio for developing the software for our numerical calculations. Errors remain our own.

†Authors' affiliations: Institute of Economics, Scuola Superiore Sant'Anna, Pisa, Italy e-mail: gdosi@sssup.it

${ }^{\ddagger}$ Department of Economics, University of Bologna, Italy

e-mail:marco.grazzi@unibo.it

${ }^{\S}$ Department of Business and Management, LUISS University, Italy

e-mail: lmarengo@luiss.it

"Department of Mathematics, Hokkaido University, Japan

e-mail: s.settepanella@math.sci.hokudai.ac.jp

(C) 2016 The Editorial Board of The Journal of Industrial Economics and John Wiley \& Sons Ltd 
emergence of the following 'stylized facts': first, wide asymmetries in productivity across firms; second, significant heterogeneity in relative input intensities even in presence of the same relative input prices; third, high intertemporal persistence in the above properties. Fourth, such heterogeneity is maintained also when increasing the level of disaggregation, thus plausibly reducing the diversity across firms' output.

The latter property has been vividly summarized by Griliches and Mairesse (1999): 'We [...] thought that one could reduce heterogeneity by going down from general mixtures as "total manufacturing" to something more coherent, such as "petroleum refining" or "the manufacture of cement." But something like Mandelbrot's fractal phenomenon seems to be at work here also: the observed variability-heterogeneity does not really decline as we cut our data finer and finer. There is a sense in which different bakeries are just as much different from each others as the steel industry is from the machinery industry.'

The bottom line is that firms operating in the same industry display a large and persistent degree of technological heterogeneity while there does not seem to be any clear sign that either the diffusion of information on different technologies, or the working of the competitive mechanism bring about any substantial reduction of such a heterogeneity, even when involving massive differences in efficiencies, as most incumbent theories would predict.

This evidence poses serious challenges not only to theory of competition and market selection, but also to any theoretical or empirical analysis which relies upon some notion of industry or sector defined as a set of production units producing under rather similar input prices with equally similar technologies, and the related notion of 'the technology' of an industry represented by means of a sectoral production function. Indeed, the aggregation conditions needed to yield the canonic production functions building from the technologies of micro entities are extremely demanding, basically involving the identity of the latter up to a constant multiplier (cf. Fisher 1965 and Hulten 2001).

Note that these problems do not only concern the neoclassical production function, whose well known properties may either not fit empirical data or fit only spuriously, ${ }^{1}$ but also non neoclassical representations of production at the industry level. If input-output coefficients à la Leontief (1986) are averages over distributions with high standard deviations and high skewness, average input coefficients may not provide a meaningful representation of the technology of that industry. Moreover, one cannot

\footnotetext{
${ }^{1}$ Shaikh (1974), for instance, shows that Cobb-Douglas production functions with constant returns to scale, neutral technological change and marginal products equal to factor rewards in presence of constant distributional shares of labour and capital (wages and profits) tend to yield a good fit to the data for purely algebraic reasons.
} 
take for granted that changes of such coefficients can be interpreted as indicators of productivity change as they may be just caused by some changes in the distribution of production among heterogeneous units, characterized by unchanged technologies.

How does one then account for the actual technology - or, better, the different techniques - in such industry? Hildenbrand (1981) suggests a direct and agnostic approach which instead of estimating some aggregate production function, offers a representation of the empirical production possibility set of an industry in the short run based on actual microdata. Each production unit is represented as a point in the input-output space whose coordinates are input requirements and output levels at full capacity. Under the sole assumptions of divisibility and additivity of production processes, ${ }^{2}$ the production possibility set is represented geometrically by the space formed by the finite sum of all the line segments linking the origin and the points representing each production unit, called a zonotope (see below). ${ }^{3}$ Hildenbrand then derives the actual 'production function' (one should more accurately say "feasible" production function) and shows that 'short-run efficient production functions do not enjoy the well-known properties which are frequently assumed in production theory. For example, constant returns to scale never prevail, the production functions are never homothetic, and the elasticities of substitution are never constant. On the other hand, the competitive factor demand and product supply functions [...] will always have definite comparative static properties which cannot be derived from the standard theory of production' (Hildenbrand, 1981, p. 1095).

In this paper we move a step forward and show that by further exploiting the properties of zonotopes it is possible to obtain rigorous measures of heterogeneity and productivity change without imposing on data a model like that implied by standard production functions. In particular, we develop a measure of productivity change that takes into consideration the entire observed production possibility set derived from observed heterogeneous production units, instead of considering only an efficient frontier. In that, as we shall discuss below, our representation of industry-level dynamics bear some complementarities to as well as differences from nonparametric estimates of (moving) efficiency frontiers (cf. Farrell 1957, Färe et al. 1994, Simar and Zelenyuk 2011).

The promise of the methodology is illustrated in this work with reference to the evidence on micro data of Italian industries and the dynamics of their distributions.

The rest of the work is organized as follows. We start with an empirical illustration of the general point (Section II). Next, Section III builds on the

\footnotetext{
${ }^{2}$ Already not entirely innocent assumptions: for a discussion cf. Dosi and Grazzi (2006).

${ }^{3}$ Note that convexity comes as a result of the chosen analytical framework and is not an assumption of an underlying theory or production (Hildenbrand, 1981, pp. 1008-9). 

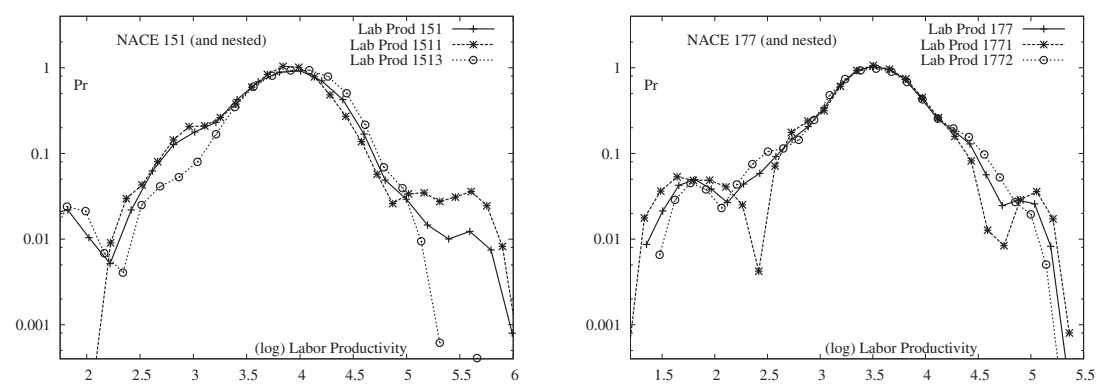

Figure 1

Empirical Distribution of Labor Productivity in 3 and 4 Digit NACE Sectors in 2006.

contribution of Hildenbrand (1981) and introduces the (normalized) volume of the zonotope as a measure of industry heterogeneity. We then propose a measure of productivity change based on the zonotope's main diagonal and we assess the role of firm entry and exit on industry level productivity growth. Section IV presents an empirical application on manufacturing firms in narrowly defined industries. Section V discusses the implications of this work and further applications of the proposed methodology.

\section{PERSISTENT MICRO HETEROGENEITY: AN ILLUSTRATION}

In order to vividly illustrate the ubiquitous, wide and persistent heterogeneity across firms within the same lines of business and in presence of roughly identical relative prices, consider two sectors of the Italian industry which one could expect not too different in terms of output, namely meat products, NACE 151, and knitted and crocheted articles, NACE 177, see Figure 1. Each of the two plots reports the empirical distribution ${ }^{4}$ of labor productivity in a three-digit NACE sector and it shows the coexistence of firms with much different levels of productivity across firms highlighting a ratio 'top to bottom' greater than 5 to 1 (in logs!). Disaggregation well reveals the 'scale freeness' of such distribution: the width of their support does not shrink if one considers the four-digit NACE sectors nested therein. The observed heterogeneity is not the result of the chosen level of industry aggregation.

Further evidence that firm-level techniques do not belong to the same 'production function' - at least of any canonic form - stems from the lack of correlation between labour productivities and 'capital productivities' (i.e. value added/capital stock). In our two foregoing sectors it is, respectively, -.02

\footnotetext{
${ }^{4}$ Densities estimates are obtained using the Epanenchnikov kernel with the bandwidth set using the optimal routine described in Silverman (1986).
} 

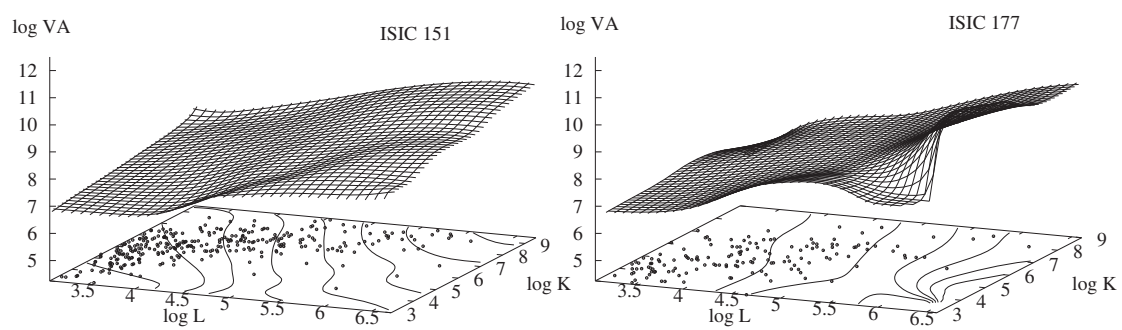

Figure 2

Adopted Techniques and Output Level in Two Different Three-Digit NACE Sectors, in 2006.

and.2, and over all the 3-digit sectors of the Italian manufacturing industry it ranges between -0.07 and. 425 with a median of. 13 .

Figure 2 graphically illustrates the point in our two sectors, with a three dimensional graph where horizontal axes represent inputs (labour and capital) and the vertical one output (value added). Using the kernel estimation techniques, smooth surfaces have been obtained from the discrete sets of observations. As a reference, the location of the observed amount of inputs $(\mathrm{l}, \mathrm{k})$ has been reported on the bottom of plots, each dot represents the input mix of a firm. The 'isoquants' report on the l-k plane the corresponding output levels. Note, first, that dots are quite dispersed over the plane and do not seem to display any regularity resembling conventional isoquant (a feature already emphasized by Hildenbrand 1981). Second, output does increase - as it should be expected - in both inputs. However, this happens in quite nonmonotonic manners: given a quantity of one input, different firms attain the same level of output with very different levels of the other input. In other words, overall degrees of efficiency seemingly widely differ.

Further, heterogeneity is very persistent over time. In our two sectors illustrations, the autocorrelation coefficients in firm-level labour productivities over a two years period rests around.8, as it does in most of the comparable 3-digit industrial sectors. Such an evidence is quite in tune with both the parametric and non-parametric estimates discussed in Bartelsman and Dhrymes (1998); Haltiwanger et al. (1999); Dosi and Grazzi (2006) among others.

All together, the evidence is robustly 'Schumpeterian' and consistent with idiosyncratic firm-level capabilities, quite inertial over time and rather hard to imitate (much more on that in Winter 2005, 2006; Nelson 2008; Dosi et al. 2008).

Granted that, how does one concisely represent the corresponding distributions of micro coefficients and their dynamics over time?

\section{ACCOUNTING FOR HETEROGENEOUS MICRO-TECHNIQUES}

Let us present a novel methodology to study production activities at the level of firms and industries which allows to account for the widespread 
TABLE 1

Production Schedules in Year 1 to 4 For an Artificial Industry

\begin{tabular}{|c|c|c|c|c|c|c|c|c|c|c|c|c|}
\hline & \multicolumn{3}{|c|}{ Year 1} & \multicolumn{3}{|c|}{ Year 2} & \multicolumn{3}{|c|}{ Year 3} & \multicolumn{3}{|c|}{ Year 4} \\
\hline & $\mathrm{L}$ & $\mathrm{K}$ & VA & $\mathrm{L}$ & $\mathrm{K}$ & VA & $\mathrm{L}$ & $\mathrm{K}$ & VA & $\mathrm{L}$ & $\mathrm{K}$ & VA \\
\hline Firm 1 & 8 & 2 & 10 & 8 & 2 & 10 & 8 & 2 & 10 & 8 & 2 & 10 \\
\hline Firm 2 & 2 & 8 & 10 & 2 & 8 & 10 & 2 & 8 & 10 & 2 & 8 & 10 \\
\hline Firm 3 & 6 & 2 & 9 & 6 & 2 & 9 & 6 & 2 & 9 & 6 & 2 & 9 \\
\hline Firm 4 & 3 & 3 & 8 & 3 & 3 & 8 & 3 & 3 & 8 & & & \\
\hline Firm 5 & 3 & 3 & 6 & 3 & 3 & 6 & 3 & 3 & 6 & 3 & 3 & 6 \\
\hline Firm 6 & 6 & 6 & 4 & 6 & 6 & 4 & & & & & & \\
\hline Firm 7 & 2 & 2 & 9 & 2 & 2 & 9 & 2 & 2 & 9 & 2 & 2 & 9 \\
\hline Firm 8 & 6 & 5 & 4 & 3 & 5 & 12 & 3 & 5 & 12 & 3 & 5 & 12 \\
\hline Firm 9 & 6 & 2 & 3 & 2 & 2 & 11 & 2 & 2 & 11 & 2 & 2 & 11 \\
\hline Firm 10 & 3 & 7 & 4 & 2 & 6 & 10 & 2 & 6 & 10 & 2 & 6 & 10 \\
\hline Industry Total & 45 & 40 & 67 & 37 & 39 & 89 & 31 & 33 & 85 & 28 & 30 & 77 \\
\hline
\end{tabular}

Notes: Number of employees (L), Capital (K) and Output (VA). External production activities in bold.

and persistent heterogeneity in productivity and production techniques, as shown in the previous section. In order to make our illustration more intuitive we develop, alongside our model, a toy example with hypothetical firms ${ }^{5}$, while an application to real data will be developed in section IV.

Without loss of generality it is possible to represent the actual technique of a production unit by means of a production activity represented by a vector (Koopmans 1977; Hildenbrand 1981)

$$
a=\left(\alpha_{1}, \ldots, \alpha_{l}, \alpha_{l+1}\right) \in \mathbb{R}_{+}^{l+1}
$$

A production unit, which is described by the vector $a$, produces during the current period $\alpha_{l+1}$ units of output by means of $\left(\alpha_{1}, \ldots, \alpha_{l}\right)$ units of input. ${ }^{6}$ Also notice that in this framework it is possible to refer to the size of the firm as to the length of vector $a$, which can be regarded as a multidimensional extension of the usual measure of firm size, often proxied either by the number of employees, sales or value added. In fact, the length of the vector allows to employ both measures of input and output in the definition of firm size.

Table 1 introduces our toy example, in which we consider a hypothetical industry containing only 10 firms. Each firm employs a given combination of labor, L, and capital, K, and produces a certain level of value added, VA, as output. We consider four years each characterized by different events. In going from year one to year two, firms 8, 9 and 10 report an unequivocal productivity increase. Then, in both years three and four one firm leaves the industry. In Table 1, vectors representing the most diverse

\footnotetext{
${ }^{5}$ Detailed instruction for replicating the toy model are available at http://mgrazzi.github.io/ zonotope_replication.html.

${ }^{6}$ Our model holds also for the multi-output case, see Appendix B for details. 


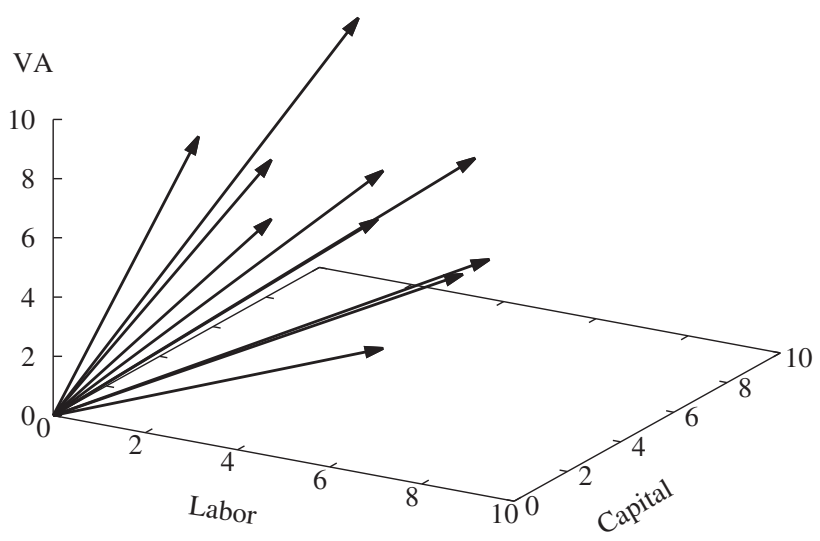

Figure 3

Vectors Representing Firms of the Toy Example in Year 1.

production techniques, which we call external vectors (more in Section C), are in bold. Figure 3 displays the 10 firms-vectors reported in Table 1.

In this framework, as noted by Hildenbrand (1981), the assumption of constant returns to scale (with respect to variable inputs) for individual production units is not necessary: indeed it is redundant if there are 'many' firms in the industry. Anyhow, the short run production possibilities of an industry with $N$ units at a given time are described by a finite family of vectors $\left\{a_{n}\right\}_{1 \leq n \leq N}$ of production activities. In order to analyze such a structure Hildenbrand introduces a short-run feasible industry production function defined by means of a Zonotope generated by the family $\left\{a_{n}\right\}_{1 \leq n \leq N}$ of production activities. More precisely let $\left\{a_{n}\right\}_{1 \leq n \leq N}$ be a collection of vectors in $\mathbb{R}^{l+1}, N \geq l+1$. To any vector $a_{n}$ we may associate a line segment

$$
\left[0, a_{n}\right]=\left\{x_{n} a_{n} \mid x_{n} \in \mathbb{R}, 0 \leq x_{n} \leq 1\right\} .
$$

Hildenbrand defines the short run total production set associated to the family $\left\{a_{n}\right\}_{1 \leq n \leq N}$ as the Minkowski sum

$$
Y=\sum_{n=1}^{N}\left[0, a_{n}\right]
$$

of line segments generated by production activities $\left\{a_{n}\right\}_{1 \leq n \leq N}$. More explicitly, it is the Zonotope

$$
Y=\left\{y \in \mathbb{R}_{+}^{l+1} \mid y=\sum_{n=1}^{N} \phi_{n} a_{n}, 0 \leq \phi_{n} \leq 1\right\} .
$$

Figure 4 displays the zonotope generated by the 10 firms in the first year of our toy example. 


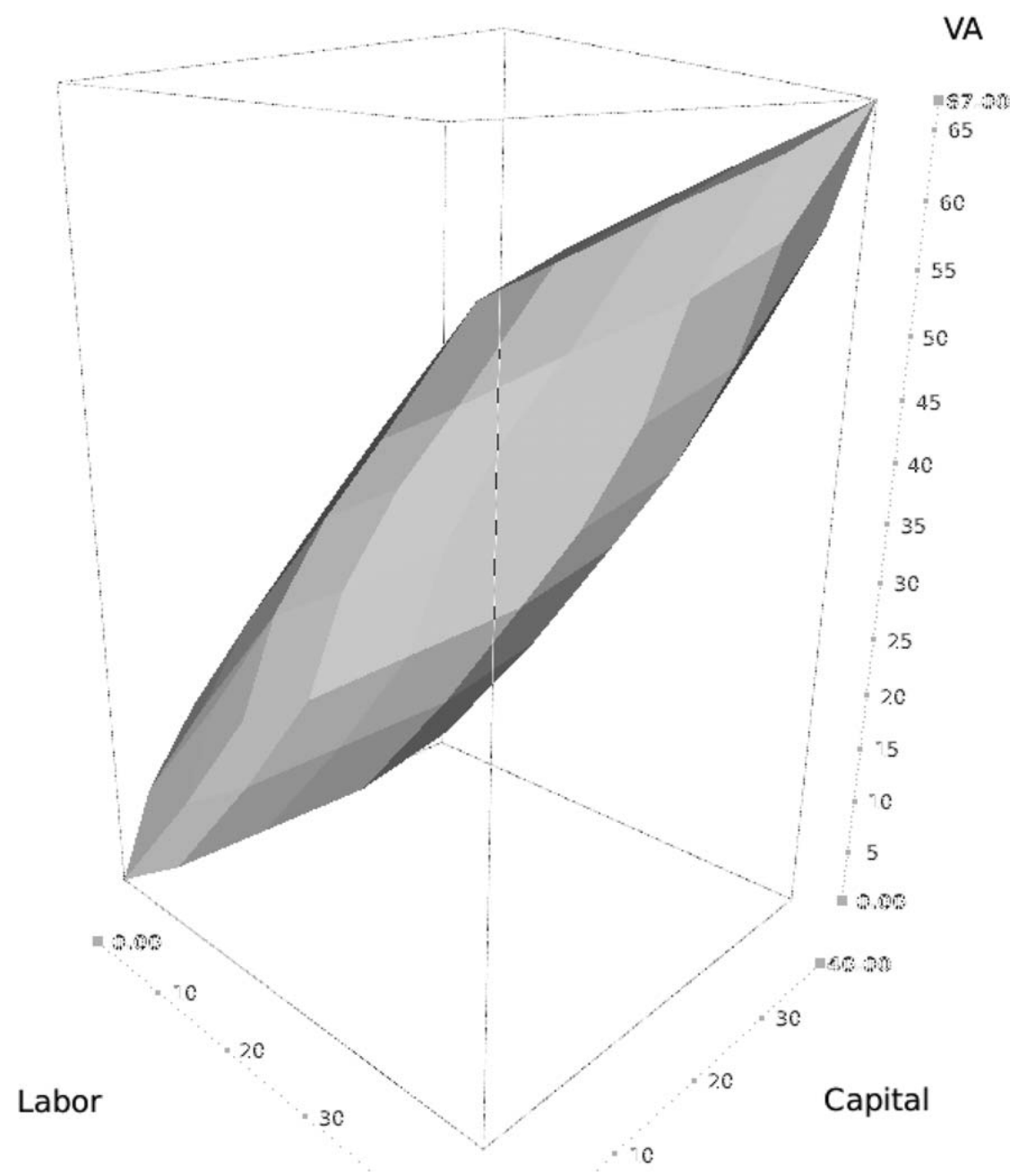

Figure 4

The Zonotope Generated by Firms of the Toy Example in Year 1.

Remark. Geometrically a Zonotope is the generalization to any dimension of a Zonohedron that is a convex polyhedron where every face is a polygon with point symmetry or, equivalently, symmetry under rotations through $180^{\circ}$. Any Zonohedron may equivalently be described as the Minkowski sum of a set of line segments in three-dimensional space, or as the three-dimensional projection of an hypercube. Hence a Zonotope is either the Minkowski sum of line segments in an l-dimensional space or the projection of an $(l+1)$-dimensional hypercube. The vectors from which the Zonotope is formed are called its generators.

${ }^{7}$ The interested reader can refer to Ziegler (1995) for a survey on Zonotopes. 
Analogously to parallelotopes and hypercubes, Zonotopes admit diagonals. We define the main diagonal of a Zonotope $Y$ as the diagonal joining the origin $O=(0, \ldots, 0) \in Y \subset \mathbb{R}^{l+1}$ with its opposite vertex in $Y$. Algebraically it is simply the sum $\sum_{n=1}^{N} a_{n}$ of all generators. That is, in our framework, each of the three coordinates denoting the upper edge of the main diagonal is given by the sum of, respectively, labor, capital and value added of the firms making up the industry. In our toy example, such coordinates are given by $(45,40,67)$.

In the following, we will denote the diagonal by $d_{Y}$ and we will call it 'production activity of the industry', as it expresses both the amount of inputs employed by the industry, and its output level.

Denote by $D$ the projection of $Y$ on the firsts $l$ coordinates, i.e.

$$
D=\left\{v \in R_{+}^{l} \mid \exists x \in \mathbb{R}_{+} \text {s.t. }(v, x) \in Y\right\}
$$

and the production function $F: D \rightarrow \mathbb{R}_{+}$associated with $Y$ as

$$
F(v)=\max \left\{x \in \mathbb{R}_{+} \mid(v, x) \in Y\right\} .
$$

In the definition above the aggregation of the various production units implies a 'frontier' associating to the level $v_{1}, \ldots, v_{l}$ of inputs for the industry the maximum total output which is obtainable by allocating, without restrictions, the amounts $v_{1}, \ldots v_{l}$ of inputs in a most efficient way over the individual production units. However, as already argued by Hildenbrand (1981) it might well be that the distribution of technological capabilities and/or the market structure and organization of the industry is such that the efficient production function could not be the focal reference either from a positive or from a normative point of view in so far as the 'frontier', first, does not offer any information on the actual technological set-up of the whole industry, and, second, does not offer any guidance as to what the industry would look like under an (unconstrained) optimal allocation of resources. This notwithstanding, estimates of the 'frontier' offer important clues on the moving best-practice opportunities and the distance of individual firms from them. Here rests also the notional complementarity between this approach and the contributions in the Data Envelopment Analysis (DEA) tradition: see Farrell (1957); Charnes et al. (1978); Daraio and Simar (2007b); Simar and Zelenyuk (2011) for major contributions in the field and Murillo-Zamorano (2004) for a review. In the DEA approach one focuses on a measure of firm's efficiency which is provided by the distance between any single firm and the efficient frontier. Conversely, in our approach, the way in which a firm contributes to industry heterogeneity depends on how such a firm combines and compares with all other firms. A similar point applies to how productivity change is measured (see below). 
The representation of any industry at any time by means of the Zonotope provides a way to assess and measure the overall degree of heterogeneity of an industry. As we shall show below, it allows also to account for the variation of production techniques adopted by firms in any industry and, at least as important, it allows to ascertain the rate and direction of productivity change.

\section{III(i). Volume of Zonotopes and heterogeneity}

Let us begin by noting that if all firms in an industry with $\mathrm{N}$ enterprises were to use the same technique in a given year, all the vectors of the associated family $\left\{a_{n}\right\}_{1 \leq n \leq N}$ of production activities would be multiples of the same vector. Hence they would lie on the same line and the generated zonotope would coincide with the diagonal $\sum_{n=1}^{N} a_{n}$, that is a degenerate zonotope of null volume. This is the case of one technology only and perfect homogeneity among firms. At the opposite extreme the maximal heterogeneity would feature an industry wherein there are firms with nearly zero inputs and other firms producing little output with a large quantity of inputs. This case of maximal heterogeneity is geometrically described by vectors that generate a zonotope which is almost a parallelotope.

In the following we provide the formula to compute the volume of the zonotope.

Let $A_{i_{1}, \ldots, i_{l+1}}$ be the matrix whose rows are vectors $\left\{a_{i_{1}}, \ldots, a_{i_{l+1}}\right\}$ and $\Delta_{i_{1}, \ldots, i_{l+1}}$ its determinant. In our framework, the first $l$ entries of each vector stand for the amount of the inputs used in the production process by each firm, while the last entry of the vector is the output. It is well known that the volume of the zonotope $Y$ in $\mathbb{R}^{l+1}$ is given by:

$$
\operatorname{Vol}(Y)=\sum_{1 \leq i_{1}<\ldots<i_{l+1} \leq N}\left|\Delta_{i_{1}, \ldots, i_{l+1}}\right|
$$

where $\left|\Delta_{i_{1}, \ldots, i_{l+1}}\right|$ is the module of the determinant $\Delta_{i_{1}, \ldots, i_{l+1}}$.

When computed in this way, the volume of the zonotope depends both on the units of measure and on the number of firms. We are therefore interested in obtaining a pure measure of the heterogeneity in techniques that allows comparisons across firms and time; that is, a measure which is independent both from the unit in which inputs and output are measured and from the number of firms making up the sector. In order to address this issue we need a way to normalize the volume of the zonotope.

The normalization we introduce is a generalization of the Gini index, which we call Gini volume of the zonotope. Analogously to the Gini index, we will consider the ratio of the volume of the zonotope $Y$ generated by the production activities $\left\{a_{n}\right\}_{1 \leq n \leq N}$ over the volume of an industry with 
TABLE 2

Volumes and Angles Accounting for Heterogeneity and Productivity Change

\begin{tabular}{lcccc}
\hline & Year 1 & Year 2 & Year 3 & Year 4 \\
\hline Vol $\left(Y^{t}\right)$ & 15217 & 12528 & 6020 & 4890 \\
$G\left(Y^{t}\right)$ & 0.1262 & 0.0975 & 0.0692 & 0.0756 \\
$G\left(\bar{Y}^{t}\right)$ & 0.1243 & 0.0940 & 0.0668 & 0.0749 \\
$G\left(Y_{e}^{t}\right)$ & 0.1555 & 0.1407 & 0.0941 & 0.0941 \\
Solid Angle & 0.4487 & 0.3009 & 0.1238 & 0.1238 \\
$G\left(Y^{t}\right) / G\left(Y_{e}^{t}\right)$ & 0.81111 & 0.6931 & 0.7358 & 0.8036 \\
$\operatorname{tg} \theta_{3}^{t}$ & 1.1128 & 1.6555 & & 1.8773 \\
$\operatorname{tg} \varphi_{1}^{t}$ & 0.8889 & 1.0540 & 1.0645 & 1.8764 \\
\hline
\end{tabular}

production activity $d_{Y}=\sum_{n=1}^{N} a_{n}$ and maximum heterogeneity. As we have already pointed out, the parallelotope is the zonotope with largest volume if the main diagonal is fixed. If $P_{Y}$ is the parallelotope of diagonal $d_{Y}$, its volume $\operatorname{Vol}\left(P_{Y}\right)$, i.e. the product of the entries of $d_{Y}$, is obviously the maximal volume that can be obtained once we fix the industry production activity $\sum_{n=1}^{N} a_{n}$, that is the total volume of an industry with production activity $d_{Y}=\sum_{n=1}^{N} a_{n}$.

Note that alike the complete inequality case in the Gini index, i.e. the case in which the index is 1 , also in our framework the complete heterogeneity case is not feasible, since in addition to firms with large values of inputs and zero output it would imply the existence of firms with zero inputs and non zero output. It has to be regarded as a limit, conceptually alike and opposite to the 0 volume case in which all techniques are equal, i.e. the vectors $\left\{a_{n}\right\}_{1 \leq n \leq N}$ are proportional and hence lie on the same line.

In what follows we consider the Gini volume defined above for the short run total production set $Y$ :

$$
G(Y)=\frac{\operatorname{Vol}(Y)}{\operatorname{Vol}\left(P_{Y}\right)} .
$$

In the first year of our toy example (Table 1 and 2), the volume of the zonotope is 15217, the volume of the parallelotope built around the zonotope's main diagonal is 120600 , so that the ratio or Gini volume, $G\left(Y^{t}\right)$, is 0.1262 . As apparent from Table 1, in year two firms 8, 9 and 10 change their production schedule and their productivity increases. The position in the input-output space of the modified firms-vectors also results in a more homogeneous industry. As reported in Table 2 the volume of the zonotope, $\operatorname{Vol}\left(Y^{t}\right)$, decreases, as well as the normalized measure of Gini 
volume, $G\left(Y^{t}\right)$. In going from period two to three, firm 6, an external vector exits the industry and further reduces heterogeneity, as $G\left(Y^{t}\right)$ decreases. In year four, firm 4 , an internal vector, exits the industry and the Gini volume signals an increase in heterogeneity. Section III(v). below investigates under what conditions firm exit (or entry) determines an increase (decrease) of heterogeneity.

\section{III(ii). Unitary production activities}

An interesting indicator of how size differences affects industry heterogeneity is provided by comparing the Gini volume $G(Y)$ of the short run total production set $Y$ with the same index computed for the zonotope $\bar{Y}$ generated by the normalized vectors $\left\{\frac{a_{n}}{\left\|a_{n}\right\|}\right\}_{1 \leq n \leq N}$, i.e. the unitary production activities. This latter Gini volume $G(\bar{Y})$ is an indicator of the heterogeneity of the industry in a hypothetical setting where all firms have the same size (norm is equal to one). Hence the only source of heterogeneity is the difference in adopted techniques, since differences in firm size do not contribute to the volume.

The comparison between the Gini volumes of the original zonotope $Y$ and of the unitary zonotope $\bar{Y}$ is informative on the relative contribution of large and small firms to the overall heterogeneity in techniques within the given industry. Intuitively, if the Gini volume $G(Y)$ of $Y$ is bigger than $G(\bar{Y})$ this means that big firms contribute to heterogeneity more than the small ones, and viceversa, if the volume $G(Y)$ is smaller than $G(\bar{Y})$. In our toy example, for instance, the Gini volume of the normalized zonotope $G(\bar{Y})$ is 0.1243 , which is smaller than 0.1262 , the value of the original Gini volume. This suggests that larger firms, i.e. companies 1 or 2 , contribute relatively more to industry heterogeneity than smaller ones.

\section{III(iii). Solid angles and external production activities}

In general, the vectors-firms making up the industry can be divided into two types according to their position in the zonotope. Some vectors contribute to form the external perimeter of the zonotope, while others lie inside it. We call the former the external vectors as they define the external zonotope $Y_{e}$. In order to provide a more formal definition of it, we need to introduce the notion of solid angle. For the ease of exposition let us start with the solid angle in a 3-dimensional space, but the idea can be easily generalized to the $(l+1)$ dimension.

In geometry, a solid angle $\Omega$ is the two-dimensional angle in threedimensional space that an object subtends at a point. It is a measure of how large the object appears to an observer looking from that point. In the International System of Units, a solid angle is a dimensionless unit of measurement called a steradian (symbol: sr). The measure of a solid angle $\Omega$ varies between 0 and $4 \pi$ steradian. 


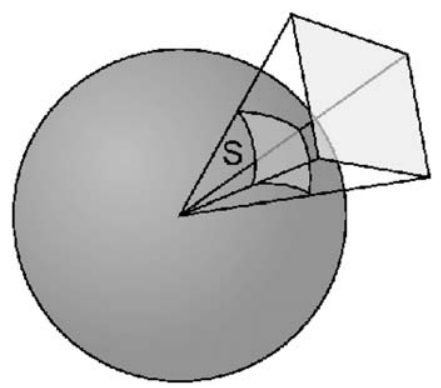

Figure 5

The Solid Angle of a Pyramid Generated by 4 Vectors.

More precisely, an object's solid angle is equal to the area of the segment of a unit sphere, centered at the angle's vertex, that the object covers, as shown in Figure 5.

In our framework, the production activities are represented by a family $\left\{a_{n}\right\}_{1 \leq n \leq N}$ of vectors. Their normalization $\left\{\frac{a_{n}}{\| a_{n} \mid}\right\}_{1 \leq n \leq N}$ will generate an arbitrary pyramid with apex in the origin. Note that, in general, not all vectors $a_{i}, i=1, \ldots, N$ will be edges of this pyramids as some vectors may lie inside the pyramid. We will call external vectors those vectors $\left\{e_{i}\right\}_{1 \leq i \leq R}$ of the family $\left\{a_{n}\right\}_{1 \leq n \leq N}$ such that their normalizations $\left\{\frac{e_{i}}{\left\|e_{i}\right\|}\right\}_{1 \leq i \leq R}$ are edges of the pyramid generated by the vectors $\left\{\frac{a_{n}}{\left\|a_{n}\right\|}\right\}_{1 \leq n \leq N}$. All the other vectors will be called internal.

This pyramid will subtend a solid angle $\Omega$, smaller or equal than $\frac{\pi}{2}$ as the entries of our vectors are all positive. We will say that the external vectors of the family $\left\{a_{n}\right\}_{1 \leq n \leq N}$ subtend the solid angle $\Omega$ if it is the angle subtended by the generated pyramid. A representation of the solid angle and of the external vectors for our illustrative example is provided in Figure 6. External production activities (i.e. firms 1, 7, 2, 10, 6, 9 clockwise) define
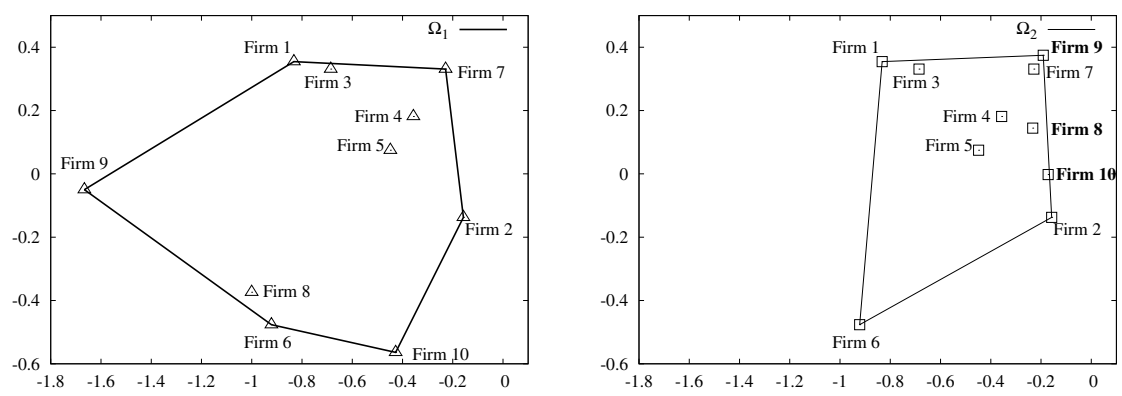

Figure 6

Planar Section of the Solid Angle Generated by All Firms of the Toy Example in Year One (left) and Two (right). The Section Plane is the One Perpendicular to the Vector Sum of Generators. 
the span of the solid angle and intuitively, they can be thought of as the analogous of the support of an empirical distribution for a single variable. Also note the variation of the solid angle captures the transition to a more homogeneous industry in going from period one to period two, as shown by Figure 6 and Table 2 .

We define the external zonotope $Y_{e}$ as the one generated by vectors $\left\{e_{i}\right\}_{1 \leq i \leq R}$. A pairwise comparison between $G\left(Y_{e}\right)$ and $G(Y)$ shows the relative importance of the density of internal activities in affecting our proposed measure of heterogeneity.

In $\mathbb{R}^{3}$ the solid angle of an arbitrary pyramid defined by the sequence of unit vectors representing edges $\left\{s_{1}, s_{2}, \ldots, s_{n}\right\}$ can be computed as

$$
\Omega=2 \pi-\arg \prod_{j=1}^{n}\left(<s_{j}, s_{j-1}><s_{j}, s_{j+1}>-<s_{j-1}, s_{j+1}>+i\left|s_{j-1} s_{j} s_{j+1}\right|\right)
$$

where parentheses $\left\langle s_{j}, s_{j-1}\right\rangle$ are scalar products, brackets $\left|s_{j-1} s_{j} s_{j+1}\right|$ are scalar triple products, i.e. determinants of the $3 \times 3$ matrices whose rows are vectors $s_{j-1}, s_{j}, s_{j+1}$, and $i$ is the imaginary unit. Indices are cycled: $s_{0}=s_{n}$ and $s_{n+1}=s_{1}$ and arg is simply the argument of a complex number.

The generalization of the definition of solid angle to higher dimensions simply needs to account for the $l$-sphere in an $l+1$-dimensional space.

\section{III(iv). Technical Change}

Let us consider a non-zero vector $v=\left(x_{1}, x_{2}, \ldots, x_{l+1}\right) \in \mathbb{R}^{l+1}$ and, for any $i \in 1, \ldots, l+1$ the projection map

$$
\begin{aligned}
p r_{-i}: & \mathbb{R}^{l+1} \rightarrow \mathbb{R}^{l} \\
& \left(x_{1}, \ldots, x_{l+1}\right) \mapsto\left(x_{1}, \ldots, x_{i-1}, x_{i+1}, \ldots, x_{l+1}\right) .
\end{aligned}
$$

Using the trigonometric formulation of Pythagoras' theorem we obtain that if $\psi_{i}$ is the angle that $v$ forms with the $x_{i}$ axis, $\theta_{i}=\frac{\pi}{2}-\psi_{i}$ is its complement and $\left\|v_{i}\right\|$ is the norm of the projection vector $v_{i}=p r_{-i}(v)$, i.e. the length of the vector $v_{i}$, then the tangent ${ }^{8}$ of $\theta_{i}$ is:

\footnotetext{
${ }^{8}$ Note that, even if the change in productivity is effectively defined by the variation of the angles $\theta_{i}$, we choose to define it using the tangent to those angles. We do this for the sake of simplicity and coherence with the 1-input and 1-output case. Indeed, the tangent is much easier to compute and, in general, the values of angles are derived by the values of the tangent. Moreover in the 1-input and 1-output case the change in productivity is simply the quotient between output and input, i.e. the tangent of the angle formed by the vector of production activity with the input axis. Notice finally that if angles increase, respectively decrease, so do their tangents.
} 


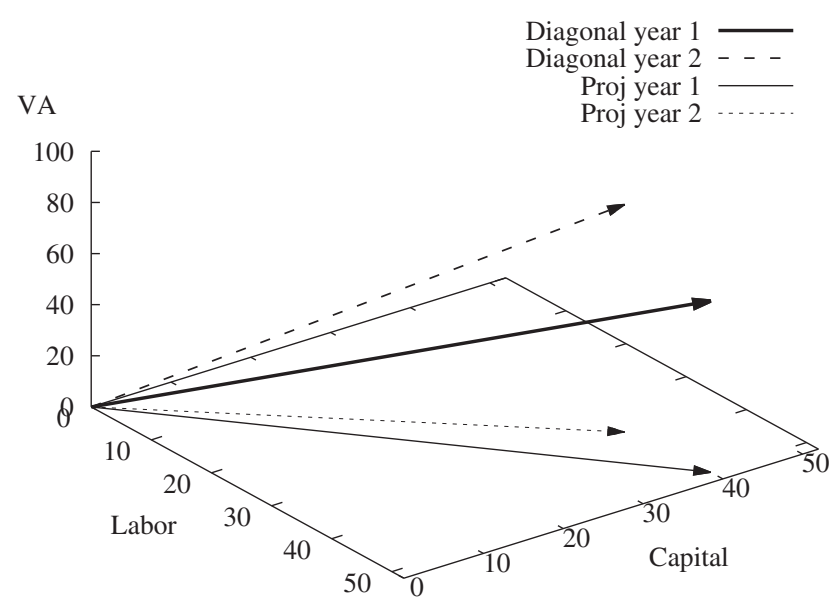

Figure 7

Productivity Increase and the Angle of the Zonotope's Main Diagonal With the Input Space in Year 1 and 2 of the Toy Example.

$$
\operatorname{tg} \theta_{i}=\frac{x_{i}}{\left\|v_{i}\right\|}
$$

In our framework we are primarily interested in the angle $\theta_{l+1}$ that the diagonal of the zonotope, i.e. the vector $d_{Y}$, forms with the space generated by all inputs, that is the $\operatorname{arctg}\left(d_{l+1} /\left\|d_{Y}\right\|\right)$ where $d_{Y}$ is equal to $d_{1}, \ldots, d_{l+1}$. Note that it is possible to think of the angle formed by $d_{Y}$ with the space generated by all inputs, as a weighted average of the productivities of all firms making up the industry, with weights given by the length of the vectors-firms. Clearly the same also applies to each individual firm, so that the angle formed by a vector-firm with the space generated by inputs expresses that firm's productivity. In this respect, the distribution of such angles can be interpreted in an analogous manner as the distribution of firms' productivities in Figure 1. Finally, this setting with two inputs and one output can easily be generalized to the case of multiple outputs, so that if we have $m$ different outputs we will consider the angles $\theta_{i}$ for $l<i \leq l+m$ (see Appendix B for more details).

In order to assess if and to what extent productivity is growing in a given industry, it is possible to analyze how the angle $\theta_{l+1}$ varies over the years. For example if the angle $\theta_{l+1}$ increases then productivity increases, because a steeper diagonal implies that the industry is able to produce more output with the same quantity of inputs. Conversely, a decrease in $\theta_{l+1}$ is a sign that productivity has decreased. Figure 7 depicts the main diagonal of the zonotope generated by the vectors-firms in our example in year 1 (solid line) and 2 (dotted line). As shown in Table 1, in year two, firms 8, 9, 
and 10 report an apparent productivity increase. Using the notation introduced above, in this setting with two inputs and one output we study the change in

$$
\operatorname{tg} \theta_{3}^{t}=\frac{d_{3}^{t}}{\left\|p r_{-3}\left(d_{Y^{t}}\right)\right\|}
$$

which is our index of productivity change, i.e. a measure of improvement in 'total factor productivity'. $\operatorname{tg} \theta_{3}^{t}$ is 1.1128 in year one and 1.6555 in year two, hence signaling the productivity increase of the industry. Then, in year three, firm 6 , a relatively inefficient one, has exited the industry and the change in $\operatorname{tg} \theta_{3}^{t}$ records another productivity increase.

Also notice that it is possible to study how the relative intensity of inputs changes over time. In order to do this, it is enough to consider the angles that the input vector, i.e. the vector with entries given by only the inputs of $d_{Y}$, forms with the different input axes. More precisely, if there are $l$ inputs and $m$ outputs and the vectors of production activities are ordered such that the first $l$ entries are inputs, then we can consider the projection function on the first $l$ coordinates:

$$
\begin{aligned}
p r_{l}: \mathbb{R}^{l+m} & \rightarrow \mathbb{R}^{l} \\
\left(x_{1}, \quad \ldots, x_{l+m}\right) & \mapsto\left(x_{1}, \ldots, x_{l}\right) .
\end{aligned}
$$

The change over time of the angle $\varphi_{i}$ between the projection vector $\operatorname{pr}\left(d_{Y}\right)$ and the $x_{i}$ axis, $1 \leq i \leq l$, captures the changes in the relative intensity of input $i$ over time with respect to all the other inputs. In our example, with only two inputs, labor and capital, and one output, we can write the relative 'intensity' of the first input (labor) as

$$
\varphi_{1}^{t}=\frac{d_{2}^{t}}{\left\|d_{1}^{t}\right\|}
$$

The increase in $\operatorname{tg} \varphi_{1}^{t}$, which is equal to 0.889 and 1.054 in year one and two, suggests that the productivity change is biased in the labor saving direction, as also apparent from Figure 7.

It is also informative to measure the changes in the normalized angles $\bar{\theta}_{i}$. Indeed, as we have done for volumes, we can consider the normalized production activities $\left\{\frac{a_{n}}{\left\|a_{n}\right\|}\right\}_{1 \leq n \leq N}$. Call $d_{\bar{Y}}$ the resulting industry production activity. Of course, one can study how it varies over time and this is equivalent to studying how the productivity of an industry changes independently from the size of the firms. In particular, the comparison of the changes of two different angles, $\theta_{i}$ and $\bar{\theta}_{i}$, reveals the relative contribution of bigger 
and smaller firms to productivity changes and, hence, on the presence of economies or diseconomies of scale.

\section{III(v). Entry and exit}

Under what circumstances does the entry of a new firm increase or decrease the heterogeneity of a given industry? In order to compute how entries and exits impact on industry heterogeneity it is enough to remark that, by the above definition of volume, given a zonotope $Z$ in the space $\mathbb{R}^{l+1}$ generated by vectors $\left\{a_{n}\right\}_{1 \leq n \leq N}$ and a vector $b=\left(x_{1}, \ldots, x_{l+1}\right) \in \mathbb{R}^{l+1}$, the volume of the new zonotope $X$ generated by $\left\{a_{n}\right\}_{1 \leq n \leq N} \cup\{b\}$ can be computed as follow:

$$
\operatorname{Vol}(X)=\operatorname{Vol}(Z)+V\left(x_{1}, \ldots, x_{l+1}\right)
$$

where $V\left(x_{1}, \ldots, x_{l+1}\right)$ is a real continuous function on $\mathbb{R}^{l+1}$ defined as:

$$
V\left(x_{1}, \ldots, x_{l+1}\right)=\sum_{1 \leq i_{1}<\ldots<i_{l} \leq N}\left|\Lambda_{i_{1}, \ldots, i_{l}}\right|,
$$

$\Lambda_{i_{1}, \ldots, i_{l}}$ being the determinant of the matrix $B_{i_{1}, \ldots, i_{l}}$ whose rows are vectors $\left\{b, a_{i_{1}}, \ldots, a_{i_{l}}\right\}$.

If $d_{Z}=\left(d_{1}, \ldots, d_{l+1}\right)$ is the diagonal of the zonotope $Z$, then the diagonal of $X$ will be $d_{X}=d_{Z}+b=\left(d_{1}+x_{1}, \ldots, d_{l+1}+x_{l+1}\right)$. The heterogeneity for the new industrial set-up will be the continuous real function

$$
G(X)=\frac{\operatorname{Vol}(Z)+V\left(x_{1}, \ldots, x_{l+1}\right)}{\operatorname{Vol}\left(P_{X}\right)}=\frac{\operatorname{Vol}(Z)+V\left(x_{1}, \ldots, x_{l+1}\right)}{\prod_{i=1}^{l+1}\left(d_{i}+x_{i}\right)}
$$

and the tangent of the angle with the input space will be the continuous real function

$$
\operatorname{tg} \theta_{l+1}\left(x_{1}, \ldots, x_{l+1}\right)=\frac{d_{l+1}+x_{l+1}}{\left\|p r_{-(l+1)}\left(d_{X}\right)\right\|}
$$

Studying the variation (i.e. gradient, hessian etc...) of these real continuous functions is equivalent to analyzing the impact of a new firm on the industry. So, for example, when these functions increase then the new firm positively contributes both to industry heterogeneity and productivity. We consider as an example the entry of a firm in the 3-dimensional case. If $Z$ is the zonotope generated by vectors $\left\{a_{n}\right\}_{1 \leq n \leq N}$ in $\mathbb{R}^{3}$ with entries $a_{n}=\left(a_{n}^{1}, a_{n}^{2}, a_{n}^{3}\right)$, the function $V\left(x_{1}, x_{2}, x_{3}\right)$ for a generic vector $b=\left(x_{1}, x_{2}, x_{3}\right)$ is 


$$
V\left(x_{1}, x_{2}, x_{3}\right)=\sum_{1 \leq i<j \leq N}\left|x_{1}\left(a_{i}^{2} a_{j}^{3}-a_{i}^{3} a_{j}^{2}\right)-x_{2}\left(a_{i}^{1} a_{j}^{3}-a_{i}^{3} a_{j}^{1}\right)+x_{3}\left(a_{i}^{1} a_{j}^{2}-a_{i}^{2} a_{j}^{1}\right)\right| .
$$

The diagonal of the new zonotope $X$ is

$$
d_{X}=\left(\sum_{i=1}^{N} a_{i}^{1}+x_{1}, \sum_{i=1}^{N} a_{i}^{2}+x_{2}, \sum_{i=1}^{N} a_{i}^{3}+x_{3}\right)
$$

We get the Gini volume for $X$ as:

$$
G(X)=\frac{\operatorname{Vol}(Z)+\sum_{1 \leq i<j \leq N}\left|x_{1}\left(a_{i}^{2} a_{j}^{3}-a_{i}^{3} a_{j}^{2}\right)-x_{2}\left(a_{i}^{1} a_{j}^{3}-a_{i}^{3} a_{j}^{1}\right)+x_{3}\left(a_{i}^{1} a_{j}^{2}-a_{i}^{2} a_{j}^{1}\right)\right|}{\sum_{i, j, k=1}^{N}\left(a_{i}^{1}+x_{1}\right)\left(a_{j}^{2}+x_{2}\right)\left(a_{k}^{3}+x_{3}\right)},
$$

where $\operatorname{Vol}(Z)$ and $\left\{a_{n}^{1}, a_{n}^{2}, a_{n}^{3}\right\}_{1 \leq n \leq N}$ are given and the tangent of the angle with the input space is:

$$
\operatorname{tg} \theta_{3}\left(x_{1}, x_{2}, x_{3}\right)=\frac{\sum_{i=1}^{N} a_{i}^{3}+x_{3}}{\sqrt{\left(\sum_{i=1}^{N} a_{i}^{1}+x_{1}\right)^{2}+\left(\sum_{i=1}^{N} a_{i}^{2}+x_{2}\right)^{2}}} .
$$

If we set the output $x_{3}$ constant or we fix the norm of $b$, i.e. the size of the firm, setting $x_{3}=\sqrt{\|b\|-x_{1}^{2}-x_{2}^{2}}$ then $G(X)$ and $\operatorname{tg} \theta_{3}\left(x_{1}, x_{2}, x_{3}\right)$ become functions in two variables, $G(X)=G(X)\left(x_{1}, x_{2}\right)$ and $\operatorname{tg} \theta_{3}\left(x_{1}, x_{2}\right)$, which can be easily studied with differential calculus.

It is important to notice that all the foregoing measures not only can be easily applied to any $l+1$-dimensional case with multi-dimensional outputs (i.e., for example, $l$ inputs and $m$ outputs in the space $\mathbb{R}^{l+m}$ ), but also to the more general case of a vector space $V$ over a field $\mathbb{K} .^{9}$

Going back to our toy example, Figure 8 shows how heterogeneity changes when a generic firm of fixed value added $(V A=5)$ enters the

${ }^{9}$ All the tools we introduced hold for any finite dimensional vector space. In that respect recall that the set $\operatorname{Hom}(V, W)$ of all linear maps between two vector spaces $V$ and $W$ over the same field $\mathbb{K}$ is a vector space itself. Thus we can consider the vector space $\mathcal{H}$ om $\left(\mathbb{R}^{l}, \mathbb{R}^{m}\right)$ in which a vector is a linear function from $\mathbb{R}^{l}$ to $\mathbb{R}^{m}$. More in general, our model applies to all finite dimensional topological vector spaces such as, for example, the space of degree $l+1$ polynomials over a field $\mathbb{K}$, the finite dimensional subspaces of smooth functions on $\mathbb{R}$ and so on. 


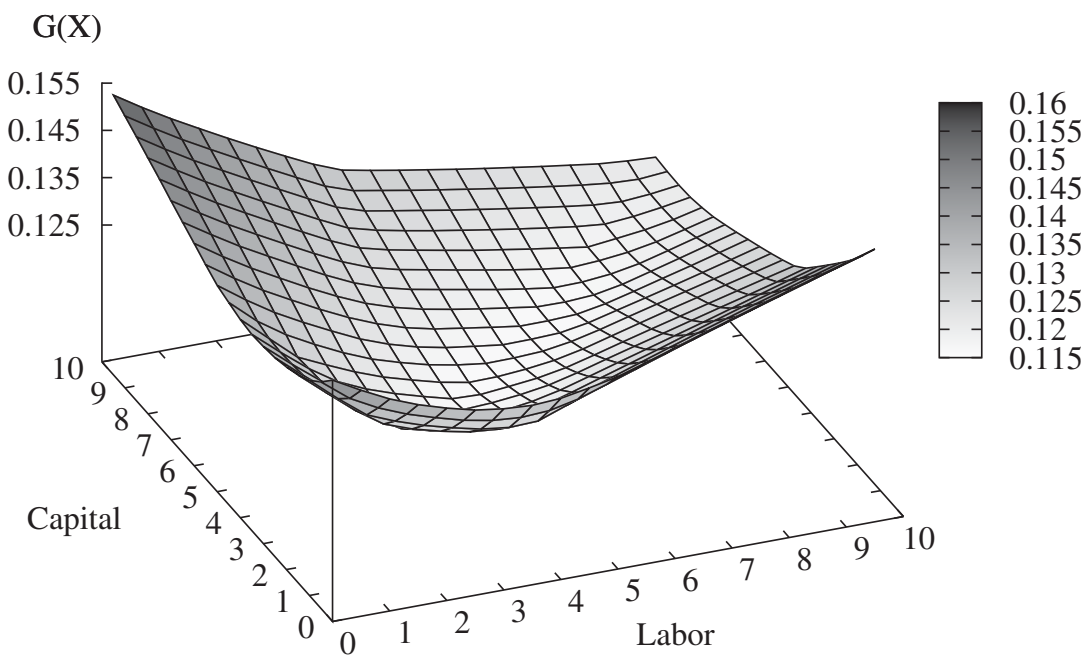

Figure 8

Variation of Heterogeneity (on the $z$ axis) When a Firm Using $x$ Units of Labor and $y$ of Units of Capital and Producing a Constant Value Added Enters the Industry.

industry in year 1 of the example. The function plotted in Fig. 8 is the function $\mathrm{G}(\mathrm{X})$ in equation (3) with $Z=Y^{1}, N=10$ and vectors $a_{n}=a_{n}^{1}$. The value on the z-axis is the degree of heterogeneity in terms of the Gini index for the different values that labour and capital might take. Analogous graphs can be plotted in order to visualize productivity change.

\section{III(vi). The Zonotope approach and other non parametric methods}

Let us consider some distinguishing features of the zonotope approach and compare it to other non-parametric methods, such as the efficient frontier approach.

The normalized volume of the zonotope, or Gini volume, provides a multidimensional measure of the heterogeneity characterizing the industry in terms of production techniques. The more the vectors-firms are spread in the inputoutput space, the bigger is the volume of the generated zonotope, and hence, the more heterogeneous the industry. Referring to our example with two inputs and one output, it is possible to have a first assessment of the firms that mostly contribute to heterogeneity by looking at the external vectors-firms (see also Table 1 and Figure 6 and 9). In year one, we find on the one hand firms 1 and 2 that are very diverse in the way they combine inputs but of comparable efficiency, and, on other hand, firms 6 and 7 that employ inputs in the same proportion, but are quite diverse in their efficiency level. Hence the diversity in both input combination and efficiency levels contributes to increasing the normalized volume of the zonotope, our measure of heterogeneity. Therefore, in 


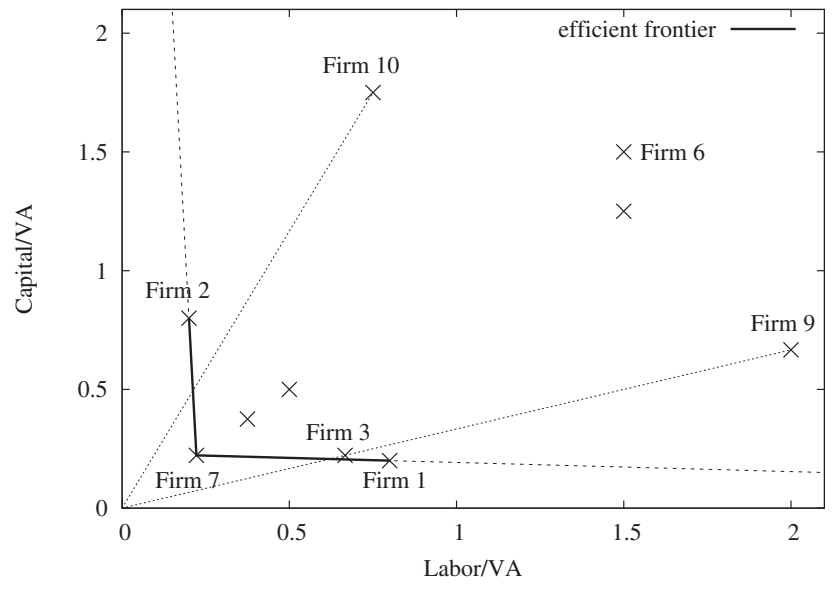

Figure 9

Data Envelopment Analysis on the Illustrative Example, Year One.

order to have a measure of cross-sectional dispersion of efficiency, that is the analog to a productivity distribution, one can refer to the distribution of firms' $\theta_{3}$, that is the angle that each vector-firm forms with the input axis.

The focus on assessing intra-industry heterogeneity as distinguished from measuring purported inefficiencies vis à vis some frontier differentiates our method from the 'efficiency frontier' approach (Farrell 1957; Charnes et al. 1978). Although both approaches are non-parametric in nature, the emphasis of the latter is on measuring firms ${ }^{10}$ inefficiency in terms of the distance from the efficient frontier, recovered by enveloping the data: the more distant a firm from the frontier, the less efficient it is. Consider, for instance, firm 10 in Figure 9 of our illustrative example which is far from the efficient frontier (solid line) or, similarly, firm 9, which employs inputs in the same proportion as firm 3, but is much less efficient.

Recent developments in efficiency analysis (Daraio and Simar 2007a) have overcome many shortcomings of the traditional deterministic approach, such as the sensitivity to measurement errors and outliers: given that all firms are compared to the frontier, a misspecification of it would heavily bias the entire analysis. In this respect robust frontiers offer a way to overcome such a problem (Cazals et al. 2002; Daraio and Simar 2005). Note, however, that our computed values for the volume (heterogeneity) and the angle (productivity change) are measures not estimates.

The focus on the frontier also enables the homonymous stream of literature to decompose productivity growth into two factors that accounts for technical change and for the change in efficiency. The former is represented

\footnotetext{
${ }^{10}$ In this stream of literature the unit of interest is called Decision Making Unit.
} 
by a shift of the frontier while the latter by the move of a firm towards the frontier. ${ }^{11}$ Clearly, in the approach that we propose, in the absence of a frontier, there is no such decomposition and all firms in the industry are considered together. Indeed, the absence of a decomposition is well in tune to a good deal of the economics of innovation literature, which emphasize the fact that all changes in technologies and in the underlying organizational routines involve in fact innovative activities - involving, to different degrees, novel discoveries, imitation, learning by doing and by using, etc (cf. the classic Nelson and Winter 1982, Rosenberg 1982 and Freeman and Soete 1997; see also Dosi and Nelson 2010). Come as it may, precisely because of these differences between the two methodologies, they are likely to offer complementary information on the state of an industry and its dynamics. In order to better appreciate the common and distinguishing features, in Appendix D we report side by side the results obtained with the zonotope approach and with frontier efficiency analysis both on the toy example and on the empirical application which we introduce in the next section. ${ }^{12}$

What the zonotope approach tells us about the revealed short-run elasticities of substitution between inputs? There is indeed a tricky measurement issue, analogous to that faced by the frontier approach where the "computation of trade-offs presents particular difficulties within DEA since the piecewise linear nature of the envelopment surfaces does not allow for unique derivatives at every point' (Rosen et al. 1998, see also Cooper et al. 2000; Greene 2008). Again, we tackle the issue following the contribution of Hildenbrand (1981) and observing that every isoquant is a polygon generated by the planar section of the zonotope. Hence, the observed elasticity of substitution is either 0 or $\infty$. It is however possible to compute the elasticity of substitution by applying smoothing techniques (Hildenbrand, 1981, pp. 1100) to the edges of the polygon. Similarly to Hildenbrand (1981) we also find values of elasticities that are rather small and vary a lot. In Appendix E we report the distribution of the elasticities of substitutions for two of the sectors investigated in the next section.

\section{AN EMPIRICAL APPLICATION}

In the following we put the methodology at work on longitudinal firmlevel data of an ensemble of Italian 4-digit industries, chosen also on the grounds of the number of observations. The dataset is based on the census of Italian firms year conducted by the Italian Statistical Office (ISTAT)

\footnotetext{
${ }^{11}$ Recent developments of the frontier approach have also focussed on the investigation of external or environmental variables, that is, variables that are neither inputs nor output under the control of the firm, but are nonetheless related to the different efficiency scores of firms (see, among the others, Daouia and Simar, 2007; Bădin et al., 2012; Mastromarco and Simar, 2015).

${ }^{12}$ Computations have been carried out using the package FEAR for R software, (Wilson 2008).
} 
TABLE 3

Sectors and Number of Observations in 1998, 2002 and 2006.

\begin{tabular}{llccc}
\hline NACE & \multicolumn{1}{c}{ Description } & 1998 & 2002 & 2006 \\
\hline 1513 & Meat and poultrymeat products & $162(7)$ & $162(10)$ & $190(9)$ \\
1721 & Cotton-type weaving & $139(9)$ & $119(11)$ & $113(7)$ \\
1772 & Knitted \& crocheted pullovers, cardigans & $137(8)$ & $117(10)$ & $100(7)$ \\
1930 & Footwear & $616(9)$ & $498(6)$ & $474(9)$ \\
2121 & Corrugated paper and paperboard & $186(7)$ & $176(9)$ & $199(11)$ \\
2222 & Printing n.e.c. & $297(11)$ & $285(10)$ & $368(8)$ \\
2522 & Plastic packing goods & $204(7)$ & $217(10)$ & $253(11)$ \\
2524 & Other plastic products & $596(9)$ & $558(9)$ & $638(10)$ \\
2661 & Concrete products for construction & $208(8)$ & $231(11)$ & $272(7)$ \\
2663 & Ready-mixed concrete & $103(8)$ & $114(8)$ & $147(10)$ \\
2751 & Casting of iron & $94(7)$ & $77(9)$ & $88(9)$ \\
2811 & Metal structures and parts of structures & $402(9)$ & $378(8)$ & $565(10)$ \\
2852 & General mechanical engineering & $473(11)$ & $511(8)$ & $825(11)$ \\
2953 & Machinery for food \& beverage processing & $131(6)$ & $134(7)$ & $159(6)$ \\
2954 & Machinery for textile, apparel \& leather & $191(10)$ & $170(10)$ & $154(12)$ \\
3611 & Chairs and seats & $205(8)$ & $201(10)$ & $229(7)$ \\
\hline
\end{tabular}

Notes: In brackets the number of external vectors in each year.

and contains information on all firms above 20 employees (Grazzi et al. 2013). Values have been deflated with the industry-specific production price index. Output is measured by value added ${ }^{13}$ at constant prices (thousands of euro), capital is proxied by (deflated) gross tangible assets (thousands of euro) and labor is simply the number of employees (full time equivalent). More details on the data set are in Appendix A. The list of sectors and the number of observations is reported in Table 3 together with the number of 'external vectors', as defined above, in brackets. ${ }^{14}$

Figure 10 reports the analog of Figure 6 for our empirical data and shows the coordinates of the normalized vectors on the unit sphere for firms making up the industry in 2002 and 2006. Both plots show that the solid angle provides a snapshot of the extreme techniques at use in a given industry. Thus, outliers matter a lot and this measure can change considerably following a variation in the adopted technique by one firm only. Hence we will not refer to the solid angle as our measure of heterogeneity, but we will focus on some normalized measures of the zonotope's volume.

\footnotetext{
${ }^{13}$ In principle our approach should use data at full capacity utilization. One should indeed represent each production unit in a $n+1$ dimensional space, where one dimension is production capacity, rather than output, and the other $n$ are the inputs needed at full capacity. This holds in Hildenbrand's 1981 paper, where he has data on maximum capacity for tankers and power plants. Unfortunately Census data do not provide this kind of information and therefore we must use output (value added) instead of capacity.

${ }^{14}$ Numerical calculations for the empirical analysis that follows as well as for the toy example above have been performed using the software 'zonohedron', written by Federico Ponchio, whom we deeply thank. More details on the software are provided in Appendix C and the software is available for download at $h t t p: / / v c g$. isti.cnr.it/ ponchio/zonohedron.php.
} 

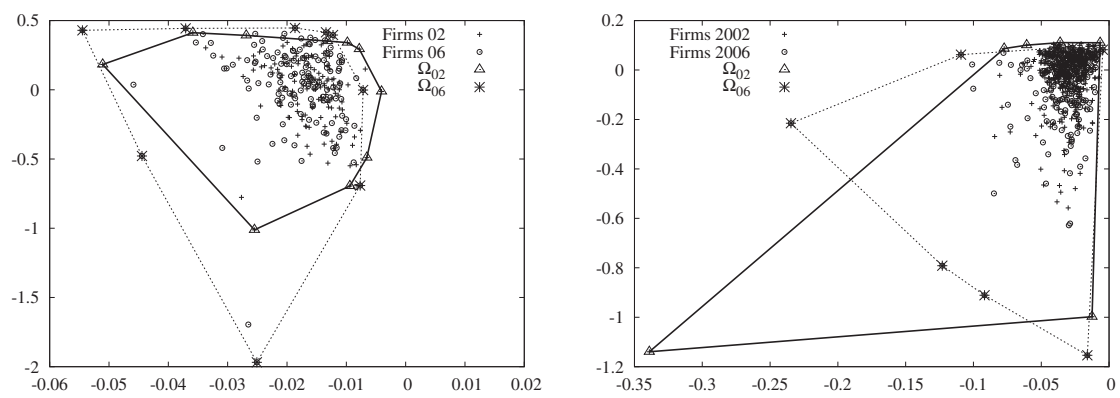

Figure 10

Planar Section of the Solid Angle Generated by All Firm-Vectors in 2002 and 2006. The Section Plane is the one Perpendicular to the Vector Sum of Generators. Meat and Poultrymeat (Sector 1513) on the Left and Footwear (Sector 1930) on the Right.

TABLE 4

Normalized Volumes in 1998, 2002 and 2006 For Selected 4 Digit Sectors.

\begin{tabular}{|c|c|c|c|c|c|c|c|c|c|}
\hline \multirow[b]{2}{*}{ NACE } & \multicolumn{3}{|c|}{ I } & \multicolumn{3}{|c|}{ II } & \multicolumn{3}{|c|}{ III } \\
\hline & & $G(Y)$ & & & $G(\bar{Y})$ & & & $G\left(Y_{e}\right)$ & \\
\hline Code & '98 & '02 & '06 & '98 & '02 & '06 & '98 & '02 & '06 \\
\hline 1513 & 0.059 & 0.051 & 0.062 & 0.082 & 0.062 & 0.096 & 0.391 & 0.201 & 0.301 \\
\hline 1721 & 0.075 & 0.068 & 0.103 & 0.075 & 0.078 & 0.124 & 0.135 & 0.120 & 0.133 \\
\hline 1772 & 0.160 & 0.122 & 0.136 & 0.154 & 0.126 & 0.130 & 0.142 & 0.273 & 0.172 \\
\hline 1930 & 0.108 & 0.139 & 0.150 & 0.110 & 0.115 & 0.123 & 0.361 & 0.562 & 0.249 \\
\hline 2121 & 0.108 & 0.043 & 0.062 & 0.081 & 0.064 & 0.081 & 0.257 & 0.105 & 0.178 \\
\hline 2222 & 0.062 & 0.077 & 0.087 & 0.077 & 0.086 & 0.115 & 0.239 & 0.328 & 0.356 \\
\hline 2522 & 0.065 & 0.061 & 0.070 & 0.071 & 0.064 & 0.074 & 0.197 & 0.261 & 0.266 \\
\hline 2524 & 0.089 & 0.083 & 0.094 & 0.097 & 0.088 & 0.096 & 0.458 & 0.269 & 0.307 \\
\hline 2661 & 0.079 & 0.088 & 0.099 & 0.100 & 0.094 & 0.110 & 0.376 & 0.234 & 0.352 \\
\hline 2663 & 0.066 & 0.067 & 0.088 & 0.111 & 0.106 & 0.111 & 0.306 & 0.192 & 0.277 \\
\hline 2751 & 0.035 & 0.037 & 0.070 & 0.064 & 0.055 & 0.073 & 0.174 & 0.107 & 0.184 \\
\hline 2811 & 0.105 & 0.109 & 0.109 & 0.117 & 0.113 & 0.122 & 0.327 & 0.480 & 0.416 \\
\hline 2852 & 0.088 & 0.102 & 0.110 & 0.100 & 0.103 & 0.111 & 0.227 & 0.395 & 0.391 \\
\hline 2953 & 0.072 & 0.095 & 0.096 & 0.098 & 0.104 & 0.111 & 0.233 & 0.155 & 0.248 \\
\hline 2954 & 0.078 & 0.074 & 0.093 & 0.086 & 0.130 & 0.113 & 0.170 & 0.141 & 0.352 \\
\hline 3611 & 0.078 & 0.099 & 0.118 & 0.107 & 0.096 & 0.121 & 0.288 & 0.233 & 0.281 \\
\hline
\end{tabular}

IV(i). Within Industry Heterogeneity and its dynamics

Table 4 reports the normalized volumes for the sectors under analysis. The first set of columns reports $G(Y)$ which is, to repeat, the ratio between the zonotope's volume and the volume of the parallelotope build on the zonotope's main diagonal, for 1998, 2002 and 2006. The volume of the cuboid (denominator) is of course much bigger than that of the zonotope (nominator) because the former is built under assumptions of maximal heterogeneity. That is why the ratio, $G(Y)$, is small in absolute value, but it signals nonetheless big differences in the production techniques employed by firms in the same industry. The dynamics over time of the ratio within 
any one industry allows to investigate how heterogeneity in the adopted techniques evolves. $G(Y)$ displays indeed an increase over time in most sectors, suggesting that heterogeneity has actually increased. ${ }^{15}$ Since $G(Y)$ is an absolute number, we can also compare this measure of heterogeneity across industries. As it might be expected, there are relevant differences in such degrees of heterogeneity, with $G(Y)$ varying in the range.03-.16. Interestingly, also sectors that are supposed to produce rather homogeneous output, such as 2661 (concrete materials) and 2663 (ready-mixed concrete) display a degree of heterogeneity comparable, if not higher, to that of the other sectors.

The second set of columns reports the value of $G(\bar{Y})$, that is the Gini volume of the unitary zonotope. As discussed in Section III, in this case the zonotope is formed by vectors having the same (unitary) length; hence firm size plays no role. For most sectors, $G(\bar{Y})$ is bigger than $G(Y)$ suggesting that, within any industry, smaller firms contribute relatively more to heterogeneity than bigger ones. In particular, in some industries, such as the Ready-mixed concrete (NACE 2663), industry heterogeneity almost doubles when all firms are rescaled to the same size. Remarkably, $G(\bar{Y})$ displays an increasing trend over time, from 2002 to 2006, pointing to growing differences in the techniques in use.

Finally, $G\left(Y_{e}\right)$ (column III) reports the Gini volume for the zonotope built on the external vectors only. As it could be expected, for all sectors $G$ $\left(Y_{e}\right)$ is bigger than $G(Y)$ as the former maps a sort of 'overall frontier' made of the best and worst techniques.

\section{IV(ii). Assessing industry level productivity changes}

Let us now analyze productivity change in our sample of Italian firms by means of the angle that the main diagonal of the zonotope forms with the input plane.

As shown in the toy illustration above, an increase in the tangent of such an angle provides evidence of an increase in the overall productivity in the industry (which innovation economists would take as an overall proxy for technical change). The first two columns of Table 5 report the rates of growth of $\operatorname{tg} \theta_{3}$ respectively for the period 1998-2002 and 2002-2006. ${ }^{16}$

Overall, not many sectors display a constant increase of productivity (i.e. increase in $\operatorname{tg} \theta_{3}^{t}$ ) in all periods. Reassuringly, the results from the method are broadly in line with the rougher evidence stemming from sheer sectorlevel average productivities and the simple observation of their micro

\footnotetext{
${ }^{15}$ This result is coherent with the evidence shown in Dosi et al. (2012) on Italian firms, although employing a different methodology to explore heterogeneity.

${ }^{16}$ Note that changing the unit of measurement, i.e. considering value added in millions (rather than thousands) of euro of course changes the value of the angle, but the variation over time - our proxy of productivity change - is not affected by the unit of measure. 
TABLE 5

Angles of the Zonotope's Main Diagonal, Rates of Growth. (A) Original Zonotope; (B) UNITARY ZONOTOPE.

\begin{tabular}{lrrrrr}
\hline NACE & \multicolumn{2}{c}{ (a) rates of growth of $\operatorname{tg} \theta_{3}$} & & \multicolumn{2}{c}{ (b) rates of growth of $\operatorname{tg} \bar{\theta}_{3}$} \\
\cline { 2 - 3 } Code & $1998-2002$ & $2002-2006$ & & $1998-2002$ & $2002-2006$ \\
\hline 1513 & -11.9073 & -11.4541 & & -3.5540 & -3.8569 \\
1721 & 10.5652 & 4.3723 & 3.6084 & 1.4179 \\
1772 & -2.5717 & 32.9763 & -0.3235 & 3.1497 \\
1930 & 3.1152 & 25.2797 & & 0.3005 & 1.9487 \\
212 & -6.8362 & -8.8206 & & -3.1977 & -4.4401 \\
222 & -23.8199 & 2.8973 & & -8.2509 & 1.0216 \\
2522 & -18.0316 & -16.8038 & & -8.2018 & -8.9011 \\
2524 & -15.2821 & 0.4118 & & -4.6589 & 0.1282 \\
2661 & 6.7277 & -18.5953 & & 1.4119 & -4.6636 \\
2663 & 25.4457 & -19.6427 & & 6.6499 & -5.9972 \\
2751 & -33.6675 & 12.9994 & -15.6436 & 5.9044 \\
281 & 6.4256 & -7.9102 & & 0.9619 & -1.2723 \\
2852 & -12.0712 & 2.1536 & & -2.4139 & 0.4289 \\
253 & 19.3637 & -4.7927 & & 1.1784 & -0.3032 \\
2954 & -0.3020 & -21.2919 & -0.0209 & -1.8708 \\
3611 & -17.9141 & 0.0892 & -2.0263 & 0.0102 \\
\hline
\end{tabular}

distribution, highlighting a widespread stagnation in Italian manufacturing over the first decade of the new millennium (cf. Dosi et al. 2012). Notice that, in tune with it, also the values computed on the unitary zonotope point to the same pattern.

The change over time of the angle $\varphi_{i}$ between the projection vector $\operatorname{pr}\left(d_{Y}\right)$ and the $x_{i}$ axis captures the changes of the quantity of input $i$ over time with respect to all the other inputs. Results are reported in Table 6. For some sectors the value of $\operatorname{tg} \varphi_{1}$ decreases over time, suggesting that

TABLE 6

Angles of the Zonotope's Main Diagonal With the Labour Input Axis. (A) Original Zonotope; (B) Unitary Zonotope.

\begin{tabular}{|c|c|c|c|c|c|c|}
\hline \multirow{2}{*}{$\frac{\text { Nace }}{\text { Code }}$} & \multicolumn{3}{|c|}{ (a) $\operatorname{tg} \varphi_{1}$} & \multicolumn{3}{|c|}{ (b) $\operatorname{tg} \bar{\varphi}_{1}$} \\
\hline & 1998 & 2002 & 2006 & 1998 & 2002 & 2006 \\
\hline 1513 & 23.0224 & 25.5508 & 27.0043 & 21.8256 & 22.6442 & 20.1068 \\
\hline 1721 & 21.0047 & 21.3726 & 18.4777 & 15.1804 & 16.0332 & 12.8776 \\
\hline 1772 & 6.8281 & 6.7041 & 5.5909 & 5.0274 & 6.2307 & 5.6227 \\
\hline 1930 & 4.5795 & 5.3113 & 5.0533 & 3.8295 & 4.5798 & 4.4675 \\
\hline 2121 & 39.0274 & 39.3436 & 40.0129 & 23.9678 & 26.6887 & 24.8705 \\
\hline 2222 & 19.1097 & 26.1785 & 24.3621 & 13.2630 & 17.2095 & 14.6962 \\
\hline 2522 & 30.2555 & 37.3270 & 43.8918 & 23.9336 & 27.9305 & 27.1886 \\
\hline 2524 & 17.9118 & 21.4862 & 19.9956 & 13.4808 & 15.7137 & 14.6993 \\
\hline 2661 & 14.1626 & 16.5427 & 17.9402 & 10.5512 & 12.3908 & 12.0912 \\
\hline 2663 & 26.9417 & 26.3218 & 26.9437 & 15.1537 & 16.5539 & 15.0585 \\
\hline 2751 & 21.8179 & 36.6899 & 31.9027 & 19.4979 & 30.2839 & 24.7586 \\
\hline 2811 & 9.1053 & 9.7865 & 9.8170 & 6.9113 & 7.8659 & 6.6393 \\
\hline 2852 & 10.0784 & 13.1988 & 13.2519 & 7.9099 & 10.4204 & 10.0850 \\
\hline 2953 & 5.4316 & 5.3541 & 5.9111 & 4.1020 & 5.0619 & 4.7180 \\
\hline 2954 & 5.0435 & 5.1530 & 5.8891 & 4.0276 & 3.7809 & 4.6053 \\
\hline 3611 & 5.7162 & 6.3274 & 6.2222 & 4.5100 & 5.3190 & 5.0401 \\
\hline
\end{tabular}


industries have moved towards relatively more labor intensive techniques (indeed a result which might reveal the peculiarities of the most recent patterns of growth, or more precisely, lack of it, of the Italian economy).

\section{CONCLUSIONS}

How does one synthetically account for the actual 'state of the technology' of an industry when firm-level techniques are widely and persistently heterogeneous?

Hildenbrand (1981) suggested a seminal methodology focusing on the geometric properties of the actual activities - that is the actual input-output relations - displayed by the firms composing the industry. He analyzed the features of such constructs in terms of the standard properties normally postulated by production functions but not born by the actual data. Here we pushed the investigation some steps further.

First, we used different measures of the volumes of the geometrical objects defined by firms' activities as measures of inter-firm technological heterogeneity.

Second, we investigated the properties of the dynamics of such objects over time as meaningful proxies for industry-level productivity change quite independent of any behavioral assumptions on allocative strategies of individual firms, and on the relationships between input prices and input intensities.

A straightforward step ahead involves indeed the study of the relationships of the foregoing dynamics with relative input prices, if any, and, probably more important, with the patterns of learning and competition characteristic of each industry.

\section{APPENDIX A: FIRM LEVEL DATA}

The database employed for the analyses, Micro.3, has been built through to the collaboration between the Italian statistical office, ISTAT, and a group of LEM researchers from the Scuola Superiore Sant'Anna, Pisa. ${ }^{17}$

Micro. 3 is largely based on the census of Italian firms yearly conducted by ISTAT and contains information on firms above 20 employees in all sectors ${ }^{18}$ of the economy for the period 1989-2006. Starting in 1998 the Census of the whole population of firms only covers companies with more than 100 employees, while in the range of employment 20-99, ISTAT directly monitors a 'rotating sample' which varies every five years. In order to complete the coverage of firms in the range of employment 20 99, Limited Liability firms have been added, subject to disclosure requirements of Italian laws. $^{19}$

\footnotetext{
${ }^{17}$ The database has been made available for work after careful censorship of individual information. More detailed information concerning the development of the database Micro. 3 are in Grazzi et al. (2013).

${ }^{18}$ In the paper we use the Statistical Classification of Economic Activities known as NACE, Revision 1.1.
} 


\section{APPENDIX B: MULTI-OUTPUT CASE}

Given a collection of vectors $\left\{a_{n}\right\}_{1 \leq n \leq N}$ in the space $\mathbb{R}^{p}, N \geq p$, the definition of Zonotope

$$
Y=\left\{y \in \mathbb{R}_{+}^{l+1} \mid y=\sum_{n=1}^{N} \phi_{n} a_{n}, 0 \leq \phi_{n} \leq 1\right\}
$$

depends equally on all entries $\left(\alpha_{1}^{n}, \ldots, \alpha_{p}^{n}\right)$ of vectors $a_{n}=\left(\alpha_{1}^{n}, \ldots, \alpha_{p}^{n}\right), 1 \leq n \leq N$. The same holds for the Volume of the Zonotope

$$
\operatorname{Vol}(Y)=\sum_{1 \leq i_{1}<\ldots<i_{p} \leq N}\left|\Delta_{i_{1}, \ldots, i_{p}}\right|
$$

where $\left|\Delta_{i_{1}, \ldots, i_{p}}\right|$ is the module of the determinant of all matrices $A_{i_{1}, \ldots, i_{p}}$ whose rows are vectors $\left\{a_{i_{1}}, \ldots, a_{i_{p}}\right\}$. Thus, the model we described in this paper naturally extends to the case where $\mathbb{R}^{p}$ is given by $\mathbb{R}^{p}=\mathbb{R}^{l} \times \mathbb{R}^{m}$, where $l$ is the number of inputs and $m>1$ is the number of outputs or $\mathbb{R}^{p}=\mathbb{R}^{p-1} \times \mathbb{R}^{1}$ where $p-1$ is the number of inputs with only 1 output.

If the number of outputs is $m>1$ then a vector $a_{n} \in \mathbb{R}^{p}$ is a $(l+m)$-tuple of entries $a_{n}=\left(\alpha_{1}^{n}, \ldots, \alpha_{l}, \alpha_{l+1}^{n}, \ldots, \alpha_{m}^{n}\right)$. Then, if $I=\left\{i_{1}, \ldots i_{j}\right\} \subset\{1, \ldots, m\}$ is a subset of indices of outputs, it is possible to consider the projection map:

$$
\begin{array}{ll}
p r_{I}: \mathbb{R}^{l+m} & \rightarrow \mathbb{R}^{l+j} \\
\left(\alpha_{1}^{n}, \ldots, \alpha_{l}, \alpha_{l+1}^{n}, \ldots, \alpha_{m}^{n}\right) & \mapsto\left(\alpha_{1}^{n}, \ldots, \alpha_{l}, \alpha_{i_{1}}^{n}, \ldots, \alpha_{i_{j}}^{n}\right) .
\end{array}
$$

The vector $\operatorname{pr}_{I}\left(a_{n}\right) \in \mathbb{R}^{l+j}$ will only have $j<m$ outputs out of the $m$ outputs of the original vector $a_{n}$. Again it is possible to perform all the computations done for the Zonotope generated by the family $\left\{a_{n}\right\}_{1 \leq n \leq N}$ for the family $\left\{\operatorname{pr}_{I}\left(a_{n}\right)\right\}_{1 \leq n \leq N}$. This allows to investigate heterogeneity with respect to only a subset of all outputs. The same applies to inputs.

Of course the concept of external vectors that we introduced in the two-inputs, one-output case (see, Section III(iii)) can easily be extended in a multi-output framework. In general, the algorithm used to compute the Volume of the Zonotope generates an explicit representation of the Zonotope itself as a set of parallelogram faces. To compute the faces the algorithm iterates over all the pair of vectors $a_{i}, a_{j}$ the following process: compute the normal $n_{i, j}$ and the sign $s_{i, j, k} \in\{0,1\}$ of the dot product of $n_{i, j}$ with each input vector $a_{k}$, zero when negative, one when positive.

The first (base) vertex of the face is computed by $\sum_{k \neq i, j} s_{i, j, k} a_{k}$, the others by adding $a_{i}, a_{j}$ and $a_{i}+a_{j}$. Once the zonohedron is in explicit form, it is very easy to identify the external vectors: i.e. those lying on the convex hull of the normalized vectors. We only need to keep track of the faces whose base vertex is the origin: in that case the pair of associated vectors $a_{i}, a_{j}$ are external. Indeed if the base vertex is the origin, all the other vertices lie on one side of the normal $n_{i, j}$ (every dot product is negative),

${ }^{19}$ Limited Liability companies (Società di Capitali) have to hand in a copy of their financial statement to the Register of Firms at the local Chamber of Commerce. 
thus that plane belong to the convex hull. This trivially extends to the multi output case. Indeed, also in this case, when we have the explicit representation of the Zonotope, the external vectors are those associated to faces with the base vertex in the origin. Of course in this case any face is defined by $n-1$ independent vectors $a_{i_{1}}, \ldots, a_{i_{n-1}}$.

Also notice that the algorithm for computing the volume of Zonotopes is polynomial on the numbers of entries $p$ of vectors, hence the computations can be performed for any large number of inputs and outputs almost as fast as the 2 inputs/ 1 output case.

In conclusion, the level of disaggregation of inputs and outputs as well as the details at which inputs and outputs are considered in the analysis are limited only by the available data.

\section{APPENDIX C: THE ALGORITHM}

This appendix illustrates the core parts of the code developed for the paper. A handson step by step guide to replicate the illustrative example is available at: http:// mgrazzi.github.io/zonotope_replication.htm.

The Gini volume is defined as the ratio between the volume of the zonohedron generated by all the input vectors and the volume of the hexahedron containing it. The diagonal of this box can be computed simply adding all the input vectors. A simple algorithm to compute the zonohedron geometry and its volume is below. The key properties used in the code are:

1. All the faces of the zonohedron are parallelograms;

2. Each pair of input vectors define a face whose normal is the cross product of the pair;

3. The vertices of the zonohedron are a weighted combination of all the input vectors with -1 or 1 coefficients;

4. A $(1,-1)$ weighted combination of vectors belongs to a face (and hence is a vertex) if the dot product of the normal of the face with each element of the sum is greater or equal to zero.

The algorithm iterates over each pair of input vectors to generate a face, compute its normal and the orientation of each vector respect to this normal. This is $O\left(n^{3}\right)$ in the number of input vectors. The volume of the zonohedron is computed adding the volume of each pyramid having for base a face and apex the origin. The code in the library is slightly more complicated by the necessity to take care of degenerate cases.

\section{Quick guide to use the software}

Zonohedron is a simple command-line program that computes a zonohedron out of a collection of $3 \mathrm{D}$ vectors. It's written in $\mathrm{C}++$ without dependencies and thus available for every platform, and can process zonohedra with thousands of generators in a short time. The program is started from the command line as such:

./zonohedron input.txt output.obj

The input file for the program is a text file with one generator vector per line:

0.3884730 .1997260 .364303

0.5191820 .1443120 .216584

0.2503670 .4450880 .391104

(C) 2016 The Editorial Board of The Journal of Industrial Economics and John Wiley \& Sons Ltd 
TABLE 7

Estimates of Order-m Efficiency Measure for the Toy Example

\begin{tabular}{lcccccccccc}
\hline Firms & 1 & 2 & 3 & 4 & 5 & 6 & 7 & 8 & 9 & 10 \\
\hline Year 1 & 1 & 1 & 1 & 1.497 & 1.495 & 2.94 & 1 & 2.47 & 0.9988 & 1.475 \\
& $(0)$ & $(0)$ & $(0)$ & $(0.035)$ & $(0.05)$ & $(0.24)$ & $(0)$ & $(0.14)$ & $(0.0177)$ & $(0.109)$ \\
Year 2 & 1 & 1 & 1 & 1.5 & 1.5 & 2.995 & 1 & 1 & 1 & 1 \\
& $(0)$ & $(0)$ & $(0)$ & $(0.0)$ & $(0.0)$ & $(0.071)$ & $(0)$ & $(0)$ & $(0)$ & $(0)$ \\
Year 3 & 1 & 1 & 1 & 1.5 & 1.5 & & 1 & 1 & 1 & 1 \\
& $(0)$ & $(0)$ & $(0)$ & $(0.0)$ & $(0.0)$ & & $(0)$ & $(0)$ & $(0)$ & $(0)$ \\
Year 4 & $(1)$ & 1 & 1 & & 1.5 & & 1 & 1 & 1 & 1 \\
& $(0)$ & $(0)$ & $(0)$ & & $(0.0)$ & & $(0)$ & $(0)$ & $(0)$ & $(0)$ \\
\hline
\end{tabular}

Notes: Standard errors in brackets.

The program saves the resulting zonohedron as a mesh in OBJ format and it also computes:

1. the volume of the zonohedra;

2. the solid angle at the origin;

3. the list of generators on the boundary of the solid angle.

For the last two computations it is required that all vectors have positive coordinates.

\section{APPENDIX D: FRONTIER ANALYSES}

In this section we present a set of results to allow the comparisons of frontier efficiency analysis ${ }^{20}$ with our proposed method. Table 7 reports for the toy example a measure of the distance of each firm from the production frontier. In particular, we consider estimates of order-m efficiency measure whose main advantage over traditional envelopment techniques is that they are more robust to extreme values (Cazals et al., 2002; Wilson, 2008). Values bigger than one suggests that the firm is not on the frontier. From the estimates of such distance function it is possible to compute the Malmiquist index for productivity change. In our toy example (see Table 1) in going from year one to year two, firms 8,9 and 10 report an unequivocal increase in productivity, this is of course captured by the Malmquist indexes which are smaller than unity for these three firms, see Table 7. In order to provide an industry-level measure of productivity change, and as standard in the literature (see, among the others Färe et al., 1994; Hsiao and Park, 2005), we consider the geometric mean of the Malmquist index for all the ten firms making up the toy example. As we consider the input orientation, we get - as expected - an industry measure smaller than one (0.654), which signals a productivity increase for the sector. For the following years it is not possible to compute any meaningful measure of variation of productivity, as the only changes are due to firms exiting the industry, and for those firms it is not possible to compute the Malmquist index.

\footnotetext{
${ }^{20} \mathrm{R}$ software and FEAR package (Wilson, 2008) have been used for the computations.
} 
TABLE 8

(A) Angles of the Zonotope's Main Diagonal, Rates of Growth. (B) MalmQUist INDEX (INPUT ORIENTATION).

\begin{tabular}{|c|c|c|c|c|}
\hline \multirow{2}{*}{$\frac{\text { Nace }}{\text { Code }}$} & \multicolumn{2}{|c|}{ (A) rates of growth of $\operatorname{tg} \theta_{3}$} & \multicolumn{2}{|c|}{ (B) Malmquist Index } \\
\hline & $1998-2002$ & $2002-2006$ & 1998-2002 & 2002-2006 \\
\hline 1513 & -10.3634 & -9.8455 & 1.04102 & 1.09689 \\
\hline 1721 & 4.0482 & 5.2562 & 0.99760 & 1.08628 \\
\hline 1772 & 1.0693 & 22.6076 & 1.13536 & 0.97827 \\
\hline 1930 & 3.7461 & 22.9161 & 0.98939 & 0.94302 \\
\hline 2121 & 3.7708 & -8.4136 & 0.98434 & 1.05707 \\
\hline 2222 & -17.6103 & 4.2627 & 1.07313 & 0.99490 \\
\hline 2522 & -14.1545 & -14.9345 & 0.96290 & 1.03422 \\
\hline 2524 & -17.6548 & -2.5974 & 1.02571 & 1.09487 \\
\hline 2661 & 1.6817 & -14.4861 & 0.88316 & 1.14890 \\
\hline 2663 & 25.9224 & -26.8413 & 0.96624 & 1.03718 \\
\hline 2751 & -24.7638 & 23.3005 & 1.14922 & 0.97883 \\
\hline 2811 & -7.2750 & -0.1386 & 0.92840 & 1.03361 \\
\hline 2852 & -8.2534 & 7.3121 & 0.95301 & 0.93898 \\
\hline 2953 & 17.8252 & -1.3819 & 0.89598 & 0.94970 \\
\hline 2954 & 6.5688 & -15.5003 & 1.03560 & 1.07015 \\
\hline 3611 & -9.2977 & 4.4400 & 0.99338 & 1.05886 \\
\hline
\end{tabular}

In Table 8 we compare our computation of productivity change to those obtained with efficiency measure. In order to run the analysis on the same set of data we 'balance' the panel of firms making up the industry in any of the two couples of years over which we investigate productivity change (1998-2002 and 2002-2006). This ensures that in the estimates of Table 8 we consider the same number of observations both in the computation of (a) our proposed measure of productivity change $\left(\operatorname{tg} \theta_{3}\right)$ as in rates of growth, and (b), equally, for the Malmquist index. The two measures are in agreement in suggesting a productivity increase (decrease) when $\operatorname{tg} \theta_{3}$ is positive (negative) and Malmquist index is smaller (bigger) than one. Note that the two measures are pretty much in accordance, and this is especially true when the productivity changes are bigger.
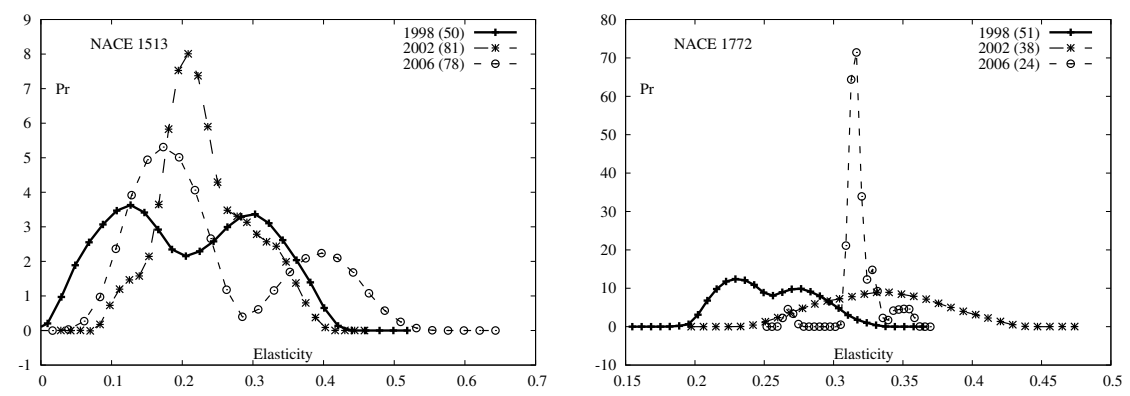

Figure 11

Distribution of Observed Elasticities of Substitution Between Capital and Labour. In Brackets the Number of Edges Over Which Elasticity is Computed. 


\section{APPENDIX E: ELASTICITIES OF SUBSTITUTION}

Figure 11 reports, for two of the industries under investigation, the distribution of the observed elasticities of substitution between capital and labour computed over the polygon which results from the planar section of the zonotope on the $50 \%$ outputisoquant, that is, half way between the origin and the upper edge of the zonotope. Elasticities of substitution are computed by applying smoothing techniques (Hildenbrand, 1981, pp. 1100) to the edges of the polygon, calculations are performed with the software zonohedron.

\section{REFERENCES}

Baily, M. N.; C. Hulten and D. Campbell, 1992, 'Productivity dynamics in manufacturing establishments,' Brookings Papers on Economic Activity: Microeconomics, 4, pp. 187-249.

Baldwin, J. R. and M. Rafiquzzaman, 1995, 'Selection versus evolutionary adaptation: Learning and post-entry performance,' International Journal of Industrial Organization, 13(4), pp. 501-522.

Bartelsman, E. J. and P. J. Dhrymes, 1998, 'Productivity dynamics: U.S. manufacturing plants, 1972-1986,' Journal of Productivity Analysis, 9(1), pp. 5-34.

Bartelsman, E. J. and M. Doms, 2000, 'Understanding productivity: Lessons from longitudinal microdata,' Journal of Economic Literature, 38(2), pp. 569-594.

Bădin, L.; C. Daraio and L. Simar, 2012, 'How to measure the impact of environmental factors in a nonparametric production model,' European Journal of Operational Research, 223(3), pp. 818-833.

Cazals, C.; J.-P. Florens and L. Simar, 2002, 'Nonparametric frontier estimation: a robust approach,' Journal of Econometrics, 106(1), pp. 1-25.

Charnes, A.; W. W. Cooper and E. Rhodes, 1978, 'Measuring the efficiency of decision making units,' European Journal of Operations Research, 2, pp. 429-444.

Cooper, W.; D. Park and P. Ciurana, 2000, 'Marginal Rates and Elasticities of Substitution with Additive Models in DEA,' Journal of Productivity Analysis, 13(2), pp. $105-123$.

Daouia, A. and L. Simar, 2007, 'Nonparametric efficiency analysis: A multivariate conditional quantile approach,' Journal of Econometrics, 140(2), pp. 375-400.

Daraio, C. and L. Simar, 2005, 'Introducing Environmental Variables in Nonparametric Frontier Models: a Probabilistic Approach,' Journal of Productivity Analysis, 24(1), pp. 93-121.

Daraio, C. and L. Simar, 2007a, Advanced Robust and Nonparametric Methods in Efficiency Analysis: methodology and applications (Springer: New York).

Daraio, C. and L. Simar, 2007b, 'Conditional nonparametric frontier models for convex and nonconvex technologies: a unifying approach,' Journal of Productivity Analysis, 28(1), pp. 13-32.

Disney, R.; J. Haskel and Y. Heden, 2003, 'Entry, exit and establishment survival in UK manufacturing,' Journal of Industrial Economics, 51(1), pp. 91-112.

Dosi, G., 2007, 'Statistical regularities in the evolution of industries. a guide through some evidence and challenges for the theory,' in F. Malerba and S. Brusoni (eds.), 'Perspectives on Innovation,' (Cambridge University Press).

Dosi, G.; M. Faillo and L. Marengo, 2008, 'Organizational capabilities, patterns of knowledge accumulation and governance structures in business firms: An introduction,' Organization Studies, 29, pp. 1165-1185. 
Dosi, G. and M. Grazzi, 2006, 'Technologies as problem-solving procedures and technologies as input-output relations: some perspectives on the theory of production,' Industrial and Corporate Change, 15(1), pp. 173-202.

Dosi, G.; M. Grazzi; C. Tomasi and A. Zeli, 2012, 'Turbulence underneath the big calm? The micro-evidence behind Italian productivity dynamics,' Small Business Economics, 39(4), pp. 1043-1067.

Dosi, G. and R. R. Nelson, 2010, 'Technological change and industrial dynamics as evolutionary processes,' in B. H. Hall and N. Rosenberg (eds.), 'Handbook of the Economics of Innovation,' chapter 4 (Elsevier: Amsterdam).

Färe, R.; S. Grosskopf; M. Norris and Z. Zhang, 1994, 'Productivity growth, technical progress, and efficiency change in industrialized countries,' American Economic Review, 84(1), pp. 66-83.

Farrell, M. J., 1957, 'The measurement of productive efficiency,' Journal of the Royal Statistical Society. Series A (General), 120(3), pp. 253-290.

Fisher, F. M., 1965, 'Embodied technical change and the existence of an aggregated capital stock,' The Review of Economic Studies, 32, pp. 326-388.

Freeman, C. and L. Soete, 1997, The Economics of Industrial Innovation (London: Pinter), 3rd edition.

Grazzi, M.; R. Sanzo; A. Secchi and A. Zeli, 2013, 'The building process of a new integrated system of business micro-data 1989-2004,' Journal of Economic and Social Measurement, 38(4), pp. 291-324.

Greene, W. H., 2008, 'The econometric approach to efficiency analysis,' in H. O. Fried; C. A. K. Lovell and S. S. Schmidt (eds.), 'The Measurement of Productive Efficiency and Productivity Change,' (Oxford: Oxford University Press).

Griliches, Z. and J. Mairesse, 1999, 'Production functions: The search for identification,' in S. Steiner (ed.), 'Econometrics and Economic Theory in the Twentieth Century: the Ragner Frisch Centennial Symposium,' (Cambridge University Press: Cambridge).

Haltiwanger, J. C.; J. I. Lane and J. R. Spletzer, 1999, 'Productivity differences across employers: The roles of employer size, age, and human capital,' American Economic Review, 89(2), pp. 94-98.

Hildenbrand, W., 1981, 'Short-run production functions based on microdata,' Econometrica, 49(5), pp. 1095-1125.

Hsiao, F. and C. Park, 2005, 'Korean and taiwanese productivity performance: Comparisons at matched manufacturing levels,' Journal of Productivity Analysis, 23(1), pp. 85-107.

Hulten, C. R., 2001, 'Total factor productivity: a short biography,' in M. H. Charles R. Hulten, Edwin Dean (ed.), 'New Developments in Productivity Analysis,' NBER Chapters, pp. 1-54 (The University of Chicago Press).

Koopmans, T. C., 1977, 'Examples of production relations based on microdata', in G. C. Harcour (ed.), 'The Microeconomic foundations of macroeconomics,' (London: Macmillan Press).

Leontief, W., 1986, Input-output economics (Oxford University Press, Oxford UK).

Mastromarco, C. and L. Simar, 2015, 'Effect of FDI and time on catching-up: new insights from a conditional nonparametric frontier analysis,' Journal of Applied Econometrics, 30(5), pp. 826-847.

Murillo-Zamorano, L. R., 2004, 'Economic efficiency and frontier techniques,' Journal of Economic Surveys, 18(1), pp. 33-77.

Nelson, R. R., 2008, 'Why do firms differ, and how does it matter? A revisitation,' Seoul Journal of Economics, 21, pp. 607-619.

Nelson, R. R. and S. G. Winter, 1982, An Evolutionary Theory of Economic Change (The Belknap Press of Harvard University Press: Cambridge, MA). 
Rosen, D.; C. Schaffnit and J. Paradi, 1998, 'Marginal Rates and Two-dimensional Level Curves in DEA,' Journal of Productivity Analysis, 9(3), pp. 205-232.

Rosenberg, N., 1982, Inside the black box: technology and economics (Cambridge University Press: Cambridge).

Shaikh, A., 1974, 'Laws of production and laws of algebra: The humbug production function,' The Review of Economics and Statistics, 56, pp. 115-120.

Silverman, B. W., 1986, Density Estimation for Statistics and Data Analysis (London: Chapman \& Hall/CRC).

Simar, L. and V. Zelenyuk, 2011, 'Stochastic FDH/DEA estimators for frontier analysis,' Journal of Productivity Analysis, 36(1), pp. 1-20.

Syverson, C., 2011, 'What determines productivity?' Journal of Economic Literature, 49(2), pp. 326-65.

Wilson, P. W., 2008, 'FEAR: A software package for frontier efficiency analysis with R,' Socio-Economic Planning Sciences, 42(4), pp. 247-254.

Winter, S. G., 2005, 'Toward an evolutionary theory of production,' in K. Dopfer (ed.), 'The Evolutionary Foundations of Economics,' pp. 223-254 (Cambridge University Press: Cambridge).

Winter, S. G., 2006, 'Toward a neo-Schumpeterian theory of the firm,' Industrial and Corporate Change, 15(1), pp. 125-141.

Ziegler, G. M., 1995, Lectures on Polytopes, Graduate Texts in Mathematics (Springer-Verlag). 